\title{
Article \\ Illuminating Host-Parasite Interaction at the Cellular and Subcellular Levels with Infrared Microspectroscopy
}

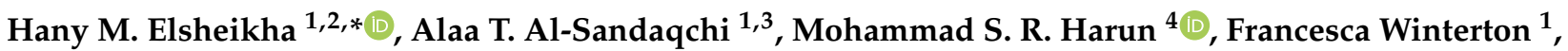 \\ Ali Altharawi ${ }^{5,6}{ }^{\circ}$, Nashwa A. Elsaied ${ }^{1}$, Carl W. Stevenson ${ }^{3}$, William MacNaughtan ${ }^{3}$, John G. M. Mina ${ }^{7}$, \\ Paul W. Denny ${ }^{7}$, Gianfelice Cinque ${ }^{8}$ (i) and Ka Lung Andrew Chan ${ }^{6, *(\mathbb{D})}$
}

check for updates

Citation: Elsheikha, H.M.; Al-Sandaqchi, A.T.; Harun, M.S.R.; Winterton, F.; Altharawi, A.; Elsaied, N.A.; Stevenson, C.W.;

MacNaughtan, W.; Mina, J.G.M.; Denny, P.W.; et al. Illuminating Host-Parasite Interaction at the Cellular and Subcellular Levels with Infrared Microspectroscopy. Cells 2022, 11, 811. https://doi.org/ 10.3390/cells11050811

Academic Editors: Tomasz P. Wrobel, Lisa Vaccari and Hugh J. Byrne

Received: 24 December 2021 Accepted: 22 February 2022 Published: 25 February 2022

Publisher's Note: MDPI stays neutral with regard to jurisdictional claims in published maps and institutional affiliations.

Copyright: (c) 2022 by the authors. Licensee MDPI, Basel, Switzerland. This article is an open access article distributed under the terms and conditions of the Creative Commons Attribution (CC BY) license (https:// creativecommons.org/licenses/by/ $4.0 /)$.
1 School of Veterinary Medicine and Science, University of Nottingham, Loughborough LE12 5RD, UK; alaa_tariq2001@yahoo.com (A.T.A.-S.); fran88@protonmail.com (F.W.); nashw95@gmail.com (N.A.E.)

2 Faculty of Veterinary Medicine, University of Liège, Sart Tilman B43, B-4000 Liège, Belgium

3 School of Biosciences, University of Nottingham, Loughborough LE12 5RD, UK; carl.stevenson@nottingham.ac.uk (C.W.S.); billmacnaughtan@gmail.com (W.M.)

4 Department of Biomedical Science, Advanced Medical \& Dental Institute, Universiti Sains Malaysia, Bertam, Kepala Batas, Pulau Pinang 13200, Malaysia; mosyamsulre@usm.my

5 Department of Pharmaceutical Chemistry, College of Pharmacy, Prince Sattam Bin Abdulaziz University, Al-Kharj 11942, Saudi Arabia; a.altharawi@psau.edu.sa

6 Institute of Pharmaceutical Science, King's College London, London SE1 9NH, UK

7 Department of Biosciences, University of Durham, Durham DH1 3LE, UK; j.g.m.mina@durham.ac.uk (J.G.M.M.); p.w.denny@durham.ac.uk (P.W.D.)

8 Diamond Light Source, Harwell Science and Innovation Campus, Didcot OX11 0DE, UK; gianfelice.cinque@diamond.ac.uk

* Correspondence: hany.elsheikha@nottingham.ac.uk (H.M.E.); ka_lung.chan@kcl.ac.uk (K.L.A.C.); Tel.: +44-0115-951-6445 (H.M.E.); +44-207-8484578 (K.L.A.C.)

Abstract: Toxoplasma gondii (T. gondii) is an opportunistic protozoan that can cause brain infection and other serious health consequences in immuno-compromised individuals. This parasite has a remarkable ability to cross biological barriers and exploit the host cell microenvironment to support its own survival and growth. Recent advances in label-free spectroscopic imaging techniques have made it possible to study biological systems at a high spatial resolution. In this study, we used conventional Fourier-transform infrared (FTIR) microspectroscopy and synchrotron-based FTIR microspectroscopy to analyze the chemical changes that are associated with infection of human brain microvascular endothelial cells (hBMECs) by T. gondii (RH) tachyzoites. Both FTIR microspectroscopic methods showed utility in revealing the chemical alterations in the infected hBMECs. Using a ZnS hemisphere device, to increase the numerical aperture, and the synchrotron source to increase the brightness, we obtained spatially resolved spectra from within a single cell. The spectra extracted from the nucleus and cytosol containing the tachyzoites were clearly distinguished. RNA sequencing analysis of T. gondii-infected and uninfected hBMECs revealed significant changes in the expression of host cell genes and pathways in response to T. gondii infection. These FTIR spectroscopic and transcriptomic findings provide significant insight into the molecular changes that occur in hBMECs during T. gondii infection.

Keywords: Toxoplasma gondii; host-pathogen interaction; blood-brain barrier; vibrational spectroscopy; synchrotron infrared microspectroscopy

\section{Introduction}

Nearly one-third of the world's human population are chronically infected by the intracellular protozoan parasite Toxoplasma gondii [1]. This protozoan can cause serious illness, especially in immunocompromised patients and in pregnant women [2,3]. T. gondii can also result in significant economic losses in production animals, particularly in sheep and goats. While some treatments are available, they are limited in scope [3]. Therefore, 
more understanding of parasite pathogenesis and host response mechanisms will lead to the development of novel therapeutics capable of protecting against disease caused by $T$. gondii infection of humans as well as many economically vital farm animal species.

How a cell responds to $T$. gondii infection and, in turn, how $T$. gondii attempts to moderate that response have been a topic of increasing interest. One such response of the parasite is to alter the host cell metabolism [4]. T. gondii is an obligate intracellular organism and auxotrophic for several key metabolites. For these reasons, T. gondii depends on the host's metabolism to leverage carbon and nutrient sources in order to sustain its growth [4]. Various analytical approaches have been used to characterize the metabolic changes that occur in host cells and tissues in response to T. gondii infection. For example, mass-spectrometry-coupled with liquid chromatography has been used to characterize the impact of $T$. gondii infection on the metabolism of cerebral cortex [5], cerebellum [5] and the whole brain in mice [6]. Nuclear magnetic resonance (NMR) was used to study the metabolic changes in human cerebrovascular endothelial cells during T. gondii infection [7]. Raman microspectroscopy has been used to characterize the spatio-temporal molecular changes in human retinal cells infected by $T$. gondii tachyzoites [4].

Vibrational infrared (IR) spectroscopy is a powerful label-free optical method that has been widely used to interrogate the chemical composition of biological materials [8]. This spectroscopic approach detects the absorbance of IR light due to molecular vibrations, thereby generating a chemical fingerprint that contains (semi)quantitative information on the constituent molecules, such as nucleic acids, proteins, lipids, and carbohydrates. Therefore, it provides an excellent opportunity to investigate biochemical changes in biological samples (tissues, cells, cell organelles) under external conditions, such as infection and drug treatment [9]. Recent advances in IR spectroscopy, including the development of cutting edge instrumentation and chemometric analytical approaches, provide more efficient tools for faster and more precise characterization of biological materials [10]. The IR spectroscopic method is often combined with optical microscopes for scanning of large areas with high spatial resolution at the cellular and sub-cellular level [11].

Synchrotron based Fourier-transform IR (FTIR) microspectroscopy has been used to characterize chemical changes in host cells under attack by T. gondii [9]. Another study has reported the use of two $\mathrm{ZnS}$ hemispheres that significantly enhance the spatial resolution and resolve subcellular features such as the nucleolus of single cells [12]. Compared to $\mathrm{CaF}_{2}$ hemispheres [13,14], the lower end of the spectral range using $\mathrm{ZnS}$ hemispheres is extended, from $1200 \mathrm{~cm}^{-1}$ to below $1000 \mathrm{~cm}^{-1}$, a significant improvement allowing the carbohydrate and DNA bands in that region to be studied [12]. This $\mathrm{ZnS}$ hemisphere device offers scope to characterize $T$. gondii interaction with host cells by FTIR at a spatial resolution that has never been observed before. To achieve subcellular spatial resolution, a recent study has shown that synchrotron IR source with a small aperture $(\sim 2.7 \mu \mathrm{m} \times 2.7 \mu \mathrm{m})$ and smaller step size $(\sim 0.9 \mu \mathrm{m})$ can produce the best imaging result in terms of image sharpness and spectral quality [15].

The aim of this study was to identify the biochemical signatures that underpin the cellular response to T. gondii infection. The combined synchrotron source with the $\mathrm{ZnS}$ hemispheres can provide the sub-cellular resolution needed for the extraction of FTIR spectra from specific regions in infected host cells. Results obtained by the $\mathrm{ZnS}$ hemisphere device and the synchrotron source were compared with data obtained using conventional FTIR microspectroscopy. In addition, high-throughput RNA sequencing (RNA-seq) technology was used to determine the transcriptome profiles and differentially expressed genes in T. gondii-infected and uninfected cells.

\section{Materials and Methods}

\subsection{Cell Lines and Culture Conditions}

Human brain microvascular endothelial cells (hBMECs) were grown in Gibco ${ }^{\circledR}$ Roswell Park Memorial Institute (RPMI) 1640 media (Thermo Fisher Scientific, Waltham, MA, USA) as described previously [9,16-19]. African Green Monkey (Cercopithecus aethiops) kidney 
epithelial (Vero) cells (ECACC, HPACC, Salisbury, UK) grown in Dulbecco's Modified Eagle Medium (DMEM) were used as surrogate host cells to sustain the growth of T. gondii tachyzoites. Both cell lines were maintained in monolayers in T75 $\left(75 \mathrm{~cm}^{2}\right)$ NUNC ${ }^{\mathrm{TM}}$ tissue culture flasks (Fisher Scientific, Leicestershire, UK) in humidified $5 \% \mathrm{CO}_{2}-95 \%$ air at $37{ }^{\circ} \mathrm{C}$. All cells used in subsequent experiments had $>99 \%$ viability as determined by the trypan blue exclusion assay.

\subsection{Parasite Culture and Purification}

Tachyzoites of T. gondii RH strain were maintained in Vero cell monolayers grown in NUNC ${ }^{\mathrm{TM}}$ T75 tissue culture flasks, as described previously [20]. The parasites were collected when lysis of at least $70 \%$ of Vero cell monolayers, due to infection, was detected. The parasite culture was maintained by inoculating the content of the infected culture flasks into new Vero cell monolayers as described previously [9,19]. To separate the tachyzoites from the host cell debris, we used PD-10 desalting columns filled with Sephadex-25 gel filtration material (GE Healthcare, Amersham, UK), as previously described [21]. Freshly purified tachyzoites were used to infect hBMECs at a multiplicity of infection (MOI) of 2 (i.e., 2 tachyzoites: 1 host cell).

\subsection{FTIR Micro Spectroscopy Analysis}

The hBMECs were seeded on $13 \mathrm{~mm} \varnothing \times 1 \mathrm{~mm}$ sterilized $\mathrm{CaF}_{2}$ windows (Crystran Ltd., Dorset, UK) positioned in the bottom of 24-well tissue culture plastic plates and incubated at $37{ }^{\circ} \mathrm{C}$ with $5 \% \mathrm{CO}_{2}$. After $24 \mathrm{~h}, \mathrm{~T}$. gondii tachyzoites were added to the cell monolayers. The infected cells were incubated for 24 and $48 \mathrm{~h}$ post infection (hpi). At each time point the medium was removed and cells (infected and control) were gently washed twice with chilled $1 \times$ Phosphate Buffered Saline (PBS; pH 7.2) to remove traces of culture medium. Cells were fixed in $4 \%$ paraformaldehyde (PFA) for 10 min followed by gentle rinsing in ultrapure water $18.2 \mathrm{M} \Omega \mathrm{cm}$ (Direct-Q $3 \mathrm{UV}$, Millipore), and air-dried for $\sim 30 \mathrm{~min}$. At least 10 independent spectra from 10 cells were recorded in each sample. FTIR measurements were performed in the transmission mode using a MCT detector fitted to an FTIR microscope (Bruker Hyperion 2000 microscope [Bruker Optics, Coventry, UK]) coupled to an FTIR spectrometer (Bruker Tensor 27 FTIR spectrometer) as described previously [19].

\subsection{Synchrotron-Based FTIR Micro Spectroscopy}

In this experiment, hBMECs $\left(1 \times 10^{4}\right)$ were seeded in 24-well tissue culture plates and incubated at $37{ }^{\circ} \mathrm{C}$ with $5 \% \mathrm{CO}_{2}$ overnight to allow the adherence and growth of the cells. Then, freshly egressed tachyzoites were used to infect hBMEC monolayers. Control hBMECs were mock-infected by medium only, without T. gondii infection. At 24 and 48 hpi, cells were harvested by trypsinization, and the trypsinized cell suspension was fixed using 4\% PFA in PBS for 10 min. Briefly, the flat surface of the hemisphere was gently rinsed using $1 \times$ PBS and fixed cell preparations were then drop-cast on three $\mathrm{ZnS}$ hemispheres per time point. The cells were allowed to dry for a few minutes followed by washing using PBS, PBS/ultrapure water and ultrapure water. Finally, two ZnS hemispheres were assembled with a thin $(6 \mu \mathrm{m})$ film of ultrapure water $(\sim 50 \mu \mathrm{L})$ sandwiched between the flat surfaces of the two hemispheres. Four cell samples were examined at each time point after infection.

The measurements were carried out at the Diamond Light Source synchrotron facility, (B22 beamline, MIRIAM). The imaging system (Hyperion 3000, Bruker Optics) includes $36 \times$ reverse Cassegrain reflective objective and condenser (Numerical aperture $=0.5$ in air, increased to 1.12 through the $\mathrm{ZnS}$ with a refractive index 2.25), a mercury cadmium telluride (MCT) liquid nitrogen cooled single element detector $50 \mu \mathrm{m}$ in size coupled with a Vertex $80 \mathrm{v}$ spectrometer. An aperture size of $\sim 2.7 \mu \mathrm{m} \times 2.7 \mu \mathrm{m}$ and a step size of $\sim 0.9 \mu \mathrm{m}$ was used at $4 \mathrm{~cm}^{-1}$ spectral resolution and 128 scans $(\sim 20 \mathrm{~s})$ per spectrum. 


\subsection{Spectral Data Processing}

FTIR images and the extracted spectra were preprocessed using OPUS software (OPUS 7.8, Bruker Optics). FTIR images were produced using the peak integration method whereby a straight baseline between a defined range was used to calculate the area under the absorbance curve. 7-10 extracted spectra from different regions of the cells ( 4 cells from each condition) were first cut to a $3000-1000 \mathrm{~cm}^{-1}$ spectral region followed by concave rubber band baseline correction with 3 iterations and 64 baseline points. Spectra were then vector normalized. Pair-wised principal component analysis (PCA) was performed on the extracted spectra using PyChem software (http:/ / pychem.sourceforge.net/; accessed on 6 December 2021) [22]. The analysis was focused on two spectral regions of interest: $3000-2800 \mathrm{~cm}^{-1}$ and $1800-1000 \mathrm{~cm}^{-1}$. Significance differences between the averaged extracted spectra from each region within the cell (control against infected) was determined using a T-test $p$ value $<0.05$ calculated using Microsoft ${ }^{\circledR}$ Excel 2010.

\subsection{Transcriptomic Analysis}

RNA-Seq was used to comprehensively study the changes in gene expression in host cells in response to T. gondii infection. Two biological replicates of control and T. gondiiinfected hBMECs at 6, 24 and 48 hpi were prepared for mRNA sequencing analysis. Briefly, the total RNA was isolated from the cell lysate of control and infected hBMECs by using a Qiagen RNeasy Plus Mini kit (Qiagen, Hilden, Germany). The quality and quantity of the extracted RNA was determined using the Agilent ${ }^{\circledR}$ RNA 6000 Nano kit and 2100 Bioanalyser (Agilent Technologies LDA UK Limited, Cheshire, UK), respectively. All samples with RQN (RNA quality number) values of 9.0 or above were processed for sequencing. Messenger RNA (mRNA) libraries were prepared using Illumina's TruSeq Stranded mRNA HT Kit [RS-122-2103] (Illumina EMEA, Cambridge, UK). Sequencing was performed as $150 \mathrm{bp}$ paired end reads on Illumina's HiSeq4000 platform (Illumina EMEA, Cambridge, UK) according to Illumina specifications. mRNA sequencing was performed by the Oxford Genomics Centre, The Wellcome Trust Centre for Human Genetics, University of Oxford, United Kingdom.

RNA-seq data analysis was carried out using VEuPathDB Galaxy Site (https:// veupathdb.globusgenomics.org/; accessed on 6 December 2021); an interactive web-based platform for large-scale data analysis. The quality of RNA sequences was checked by FastQC Galaxy version 0.11.3. Trimmomatic Galaxy Version 0.36 .5 ) and low-quality reads (default settings) and adapters (ILLUMINACLIP-trim standard adapter sequence for paired ended for MiSeq and HiSeq sequencers) were removed. The cleaned reads were aligned to the HostDB-52_HsapiensREF_Genome using HISAT2 (Galaxy Version 2.0.5). The read counting was performed using HTSeq-count (Galaxy Version HTSeq: default; SAMTOOLS: 1.2; PICARD: 1.134). We analyzed the differential gene expression level between infected and control samples by using EdgeR (Galaxy Version 1.0.0), with filtered counts used as input [23]. The individual gene expression was calculated as the mean expression of each gene averaged over all samples of each group and presented as the logarithm of counts per million reads. The false-discovery rate (FDR) and adjusted $p$ value were used for multiple test comparisons according to the Benjamini-Hochberg procedure. We used a threshold (adjusted $p$-value/FDR value $<0.05$ and absolute $\log _{2} \mathrm{FC} \geq 1$ or $\leq-1$ ) to identify the differentially expressed genes (DEGs). PCA, hierarchical clustering heatmaps and volcano blots of the DEGs at all time points and at each time point post-infection were produced by using PCA plot w ggplot2 (Galaxy Version 2.2.1), heatmap2 (Galaxy Version 3.0.1), and R Studio (version 4.1.1) with ggplot2 package for visualization. To identify the biological relevance of the DEGs, pathway analysis was performed using the REACTOME pathway browser (https://reactome.org/PathwayBrowser/; accessed on 13 December 2021) and single-sample gene set enrichment analysis (ssGSEA) to obtain pathway expression values [24]. 


\section{Results}

\subsection{Single Cell Analysis Using Conventional FTIR Microspectroscopy}

The spectra and PCA results obtained from the single cell analysis using a conventional FTIR microscopy without synchrotron or the hemispherical $\mathrm{ZnS}$ lenses approach are shown in Figure 1. These results are based on the averaged FTIR absorbance from the individual hBMECs and therefore it was not possible to reveal subcellular differences between the infected and the control cells. Figure 1A,B show that the lipid content is slightly lowered $24 \mathrm{hpi}$ but was increased at $48 \mathrm{hpi}$. The results of PCA at 24 and 48 hpi (Figure 1C,D) revealed insignificant differences between the control and infected cells from the first principal component (PC1). However, significant differences were observed in PC2 with changes mainly associated with the lipid $\mathrm{CH}$ stretching bands and protein amide I and II. Cells infected for $24 \mathrm{~h}$ had an overall lower lipid (negative $v(\mathrm{CH})$ bands) in the loading vector, however at $48 \mathrm{hpi}$, the lipid content was higher in the infected cells, in agreement with the results shown in Figure 1A,B.

\subsection{Subcellular Mapping Using Synchrotron MicroFTIR and ZnS Hemispheres}

We applied the newly developed hemispherical ZnS lenses to increase the spatial resolution of FTIR imaging so that spectra from nucleus and parasite-containing cytosol regions in single infected cells could be discerned. We also compared the spectral difference between the cytosol of uninfected cells in control culture, uninfected cells in infected culture, and infected cells as a function of infection time. A typical spectrum extracted from the imaging measurements is shown in Figure 2, highlighting the high quality of spectrum obtained despite the small aperture used.

Different bands were used to produce FTIR images that represent the different subcellular areas within a single cell. The overall outline of the cell was marked by the protein amide II (1593-1496 $\left.\mathrm{cm}^{-1}\right)$ and phosphate $v_{\text {sym }}\left(\mathrm{PO}_{2}\right)^{-}\left(1102-1065 \mathrm{~cm}^{-1}\right)$ bands; lipid $v_{\text {asym }}\left(\mathrm{CH}_{2}\right)\left(2946-2908 \mathrm{~cm}^{-1}\right)$ and $v(\mathrm{C}=\mathrm{O})\left(1763-1718 \mathrm{~cm}^{-1}\right)$ bands were used to highlight the lipid-rich cytosol region, $v_{\mathrm{sym}}\left(\mathrm{CH}_{3}\right)\left(2885-2865 \mathrm{~cm}^{-1}\right)$; while the $v(\mathrm{C}=\mathrm{O})(1730-1707)$ band was used to reveal the area of the nucleus [25]. Cells were measured in their hydrated state (in aqueous environment) to avoid large differences in the microenvironment of analyzed cells. For example, dried nucleic acid can lead to significantly different spectral bands compared to their hydrated counterpart [26]. As a result, amide I at $\sim 1656 \mathrm{~cm}^{-1}$ was not used in the image generation because higher noise/saturation was observed in spectral regions where water has strong absorbances $\left(>3000 \mathrm{~cm}^{-1}\right.$ and $\left.1680-1620 \mathrm{~cm}^{-1}\right)$.

A representative set of FTIR maps of infected and control cells at 24 and $48 \mathrm{~h}$ after infection are shown in Figure 3. The images of all analyzed cells are shown in Figures S1-S4. The amide II and $v_{\mathrm{sym}}\left(\mathrm{PO}_{2}\right)^{-}$peak integration produced FTIR maps showing the overall shape of the cell, which correlated well with the visible images. The FTIR maps from the two lipid bands $v_{\text {asym }}\left(\mathrm{CH}_{2}\right)$ and $v(\mathrm{C}=\mathrm{O})$ showed uneven lipid distribution within the single cell and appeared to be broadly complementary to the FTIR maps of the DNA $v_{\text {sym }}\left(\mathrm{CH}_{3}\right)$ and $v(\mathrm{C}=\mathrm{O})$ bands, which indicated the nuclear region of the cell. Whilst the SR-FTIR approach revealed the distribution of various subcellular components inside a single cell, no obvious differences between the infected and control cells were observed. However, the high spatial resolution approach allowed spectra from different regions of a single cell to be dissociated. The spectra extracted from lipid-rich and lipid-poor cytosol, and nucleus regions were compared using pair-wised PCA to reveal the spectroscopic difference between the control and infected cells. The results of the PCA are shown in Figures 4 and 5 . 
A

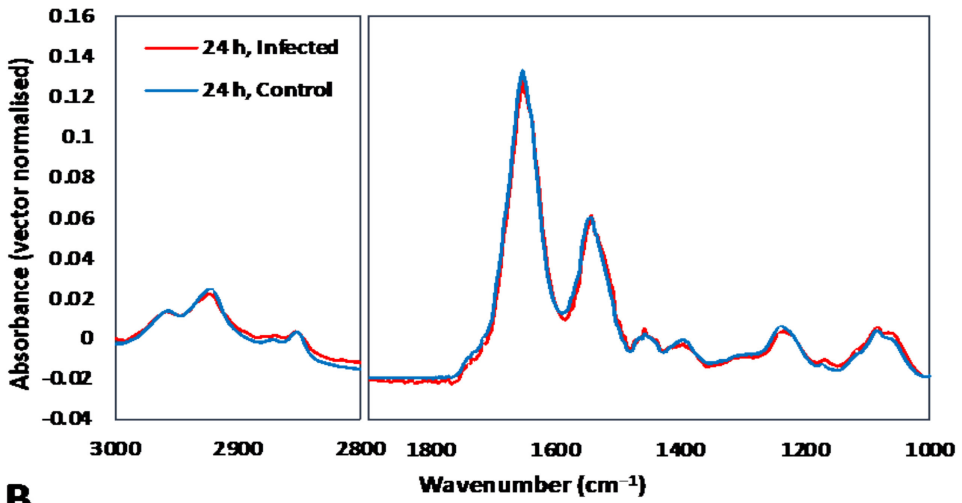

B

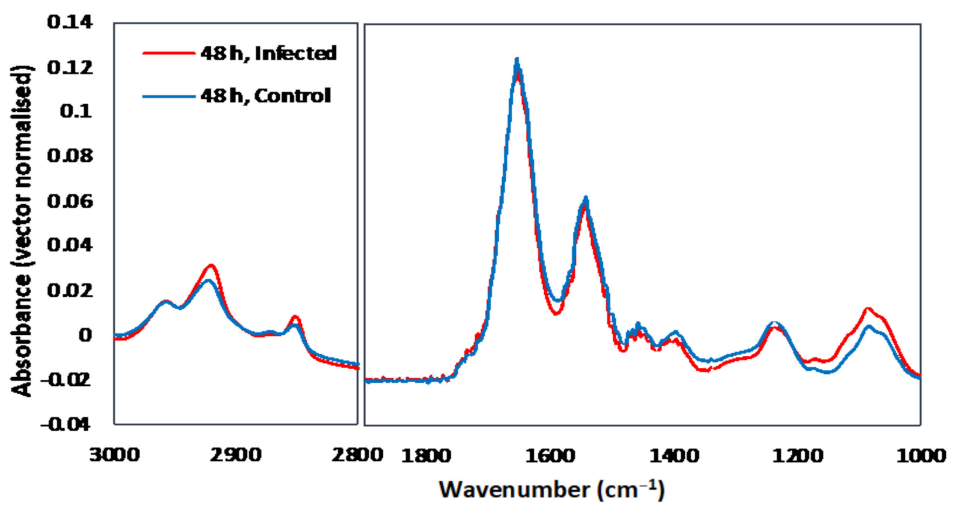

C

$24 \mathrm{~h}$ treatment

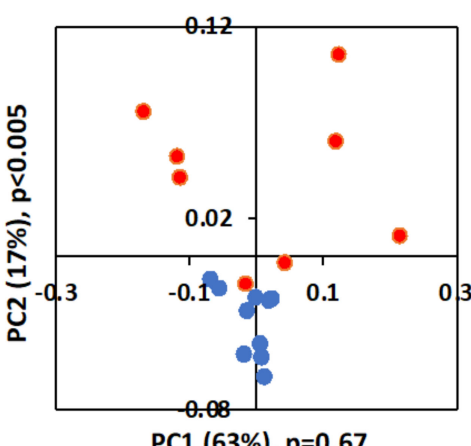

PC1 $(63 \%), p=0.67$
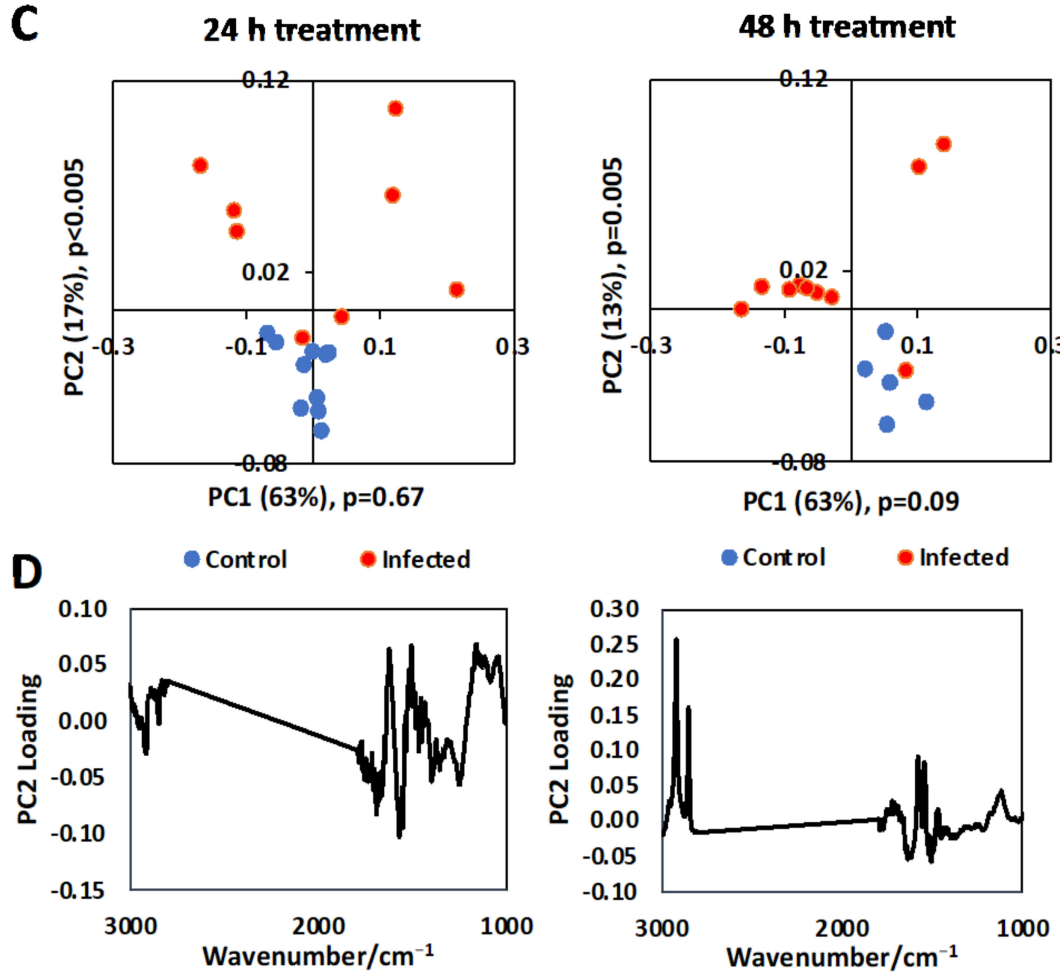

Figure 1. The averaged spectra of cells at $24 \mathrm{~h} \mathrm{(A)} \mathrm{and} 48 \mathrm{~h}$ (B) after treatment. Pair-wised (control versus infected) PCA of spectral data obtained from traditional FTIR microscopy at $24 \mathrm{~h}$ and $48 \mathrm{~h}$ after infection with (C) showing the score scatter plots and (D) showing the PC2 loading vectors. 


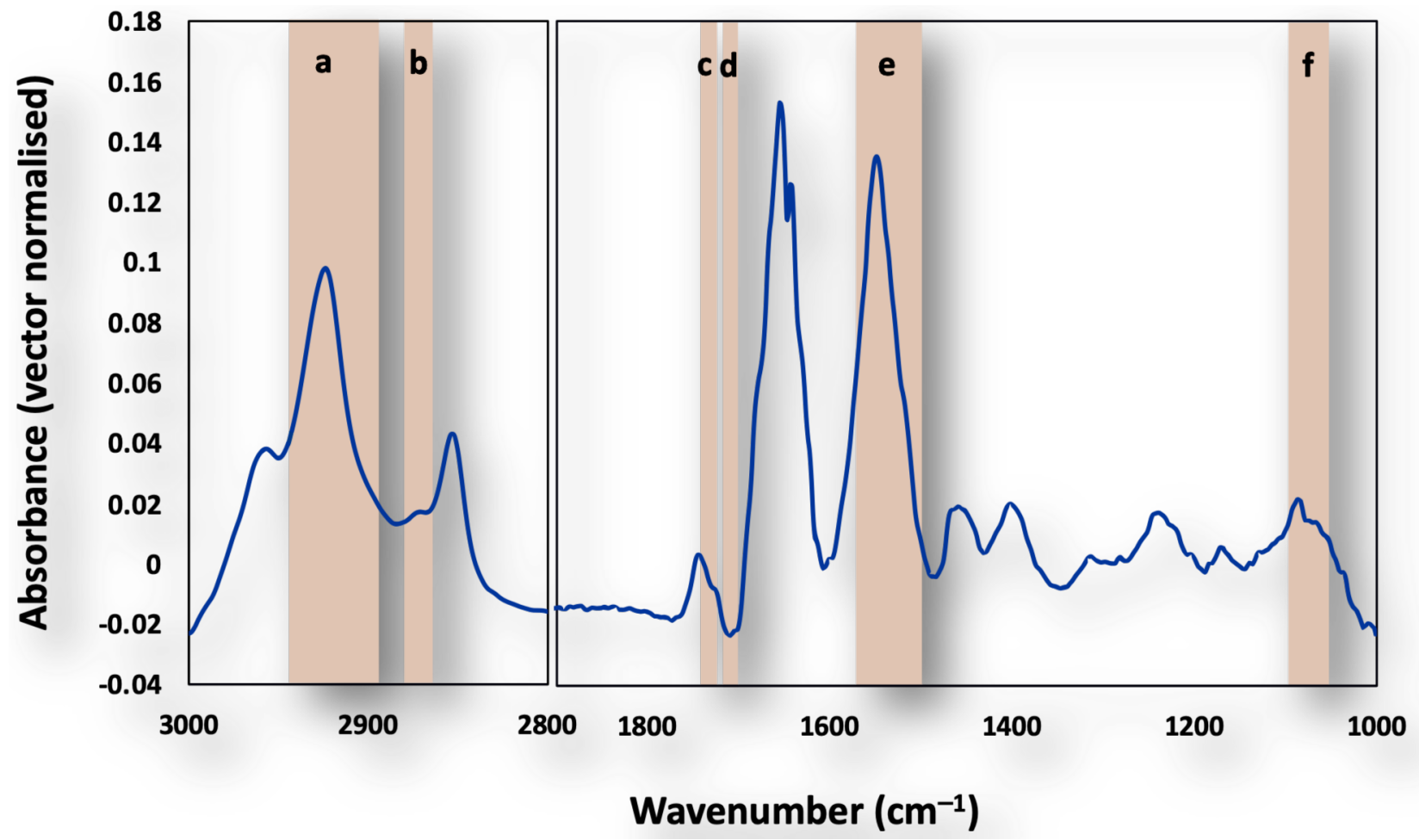

Figure 2. Typical SR-FTIR spectrum (3000-2800 $\mathrm{cm}^{-1}$ and $1800-1000 \mathrm{~cm}^{-1}$ ) of the cell measured through the $\mathrm{ZnS}$ lenses with peaks a-f used to generate FTIR images of (a) lipid $v_{\text {asym }}\left(\mathrm{CH}_{2}\right)$ (2946-2908 $\left.\mathrm{cm}^{-1}\right)$, (b) DNA $v_{\text {sym }}\left(\mathrm{CH}_{3}\right)\left(2885-2865 \mathrm{~cm}^{-1}\right)$, (c) lipid $v(\mathrm{C}=\mathrm{O})\left(1763-1718 \mathrm{~cm}^{-1}\right),(\mathrm{d})$ DNA $\vee(\mathrm{C}=\mathrm{O})\left(1730-1707 \mathrm{~cm}^{-1}\right),(\mathrm{e})$ protein amide II $\left(1593-1496 \mathrm{~cm}^{-1}\right)$ and (f) phosphate $v_{\mathrm{sym}}\left(\mathrm{PO}_{2}\right)^{-}$ (1102-1065 $\left.\mathrm{cm}^{-1}\right)$, respectively.

Figure 4 shows the pair-wised comparison between the control and infected cells at 24 hpi. There is a separation in PC1 (the most important PC) from the lipid-rich cytosol region, but the separation was not significant $(p>0.05)$ and not in any of the other pairwised (control versus infected) comparison PCs. The large range of PC1 score in the infected cell has shown that the infected cells were highly variable in the lipid-rich domain, suggesting a heterogeneous response among the infected cells at the early stage of infection. In the lipid-poor region of the cytosol, PC1 has shown a significant difference between control and infected cells with changes mainly found in the protein amide I and II peaks. In the nucleus region, PC1 did not show significant difference $(p>0.05)$ between the control and infected cells. However, there was a significant difference from PC2 (the second most important PC) with the loading vector highlighting positive bands for the protein amide I, II and III and negative bands for the lipid $v\left(\mathrm{CH}_{2}\right)$ and $v(\mathrm{C}=\mathrm{O})$ bands, suggesting a higher protein and lower lipid content in the nucleus of the control when compared to the infected cell at $24 \mathrm{hpi}$. This result is consistent with the result obtained by conventional FTIR microspectroscopy.

Figure 5 shows the results at $48 \mathrm{hpi}$. In contrast to $24 \mathrm{~h}$, all pair-wised comparisons between control and infected cells showed significant differences $(p<0.05)$ in PC1, suggesting that longer infection time produced more marked effect on the infected cells. Changes in the cytosol, whether lipid-rich or lipid-poor, produced a similar difference as shown by the similarity in their PCA loading plots. The infected cells showed an increase in lipid $v\left(\mathrm{CH}_{2}\right)$ and $v(\mathrm{C}=\mathrm{O})$ bands. The nucleus region also showed an increase in lipid in the infected cell with rather complex changes in the protein, phosphate, and nucleic acid regions, suggesting several biochemical changes have occurred. 

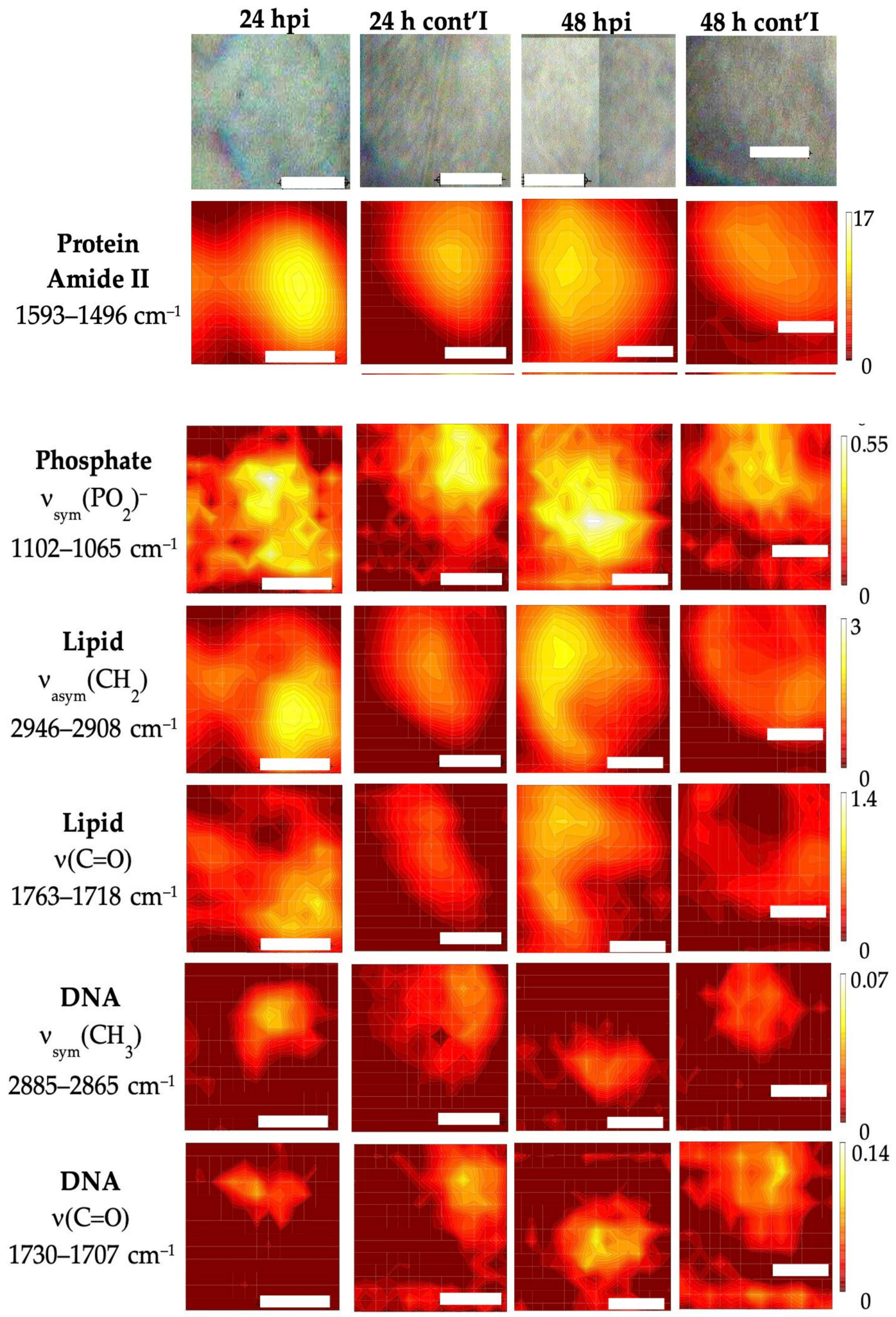

Figure 3. A representative set of visible images (top row) and FTIR images (integration range shown on the left column) of infected and control cells at 24 and $48 \mathrm{hpi}$. Scale bars $=10 \mu \mathrm{m}$. 

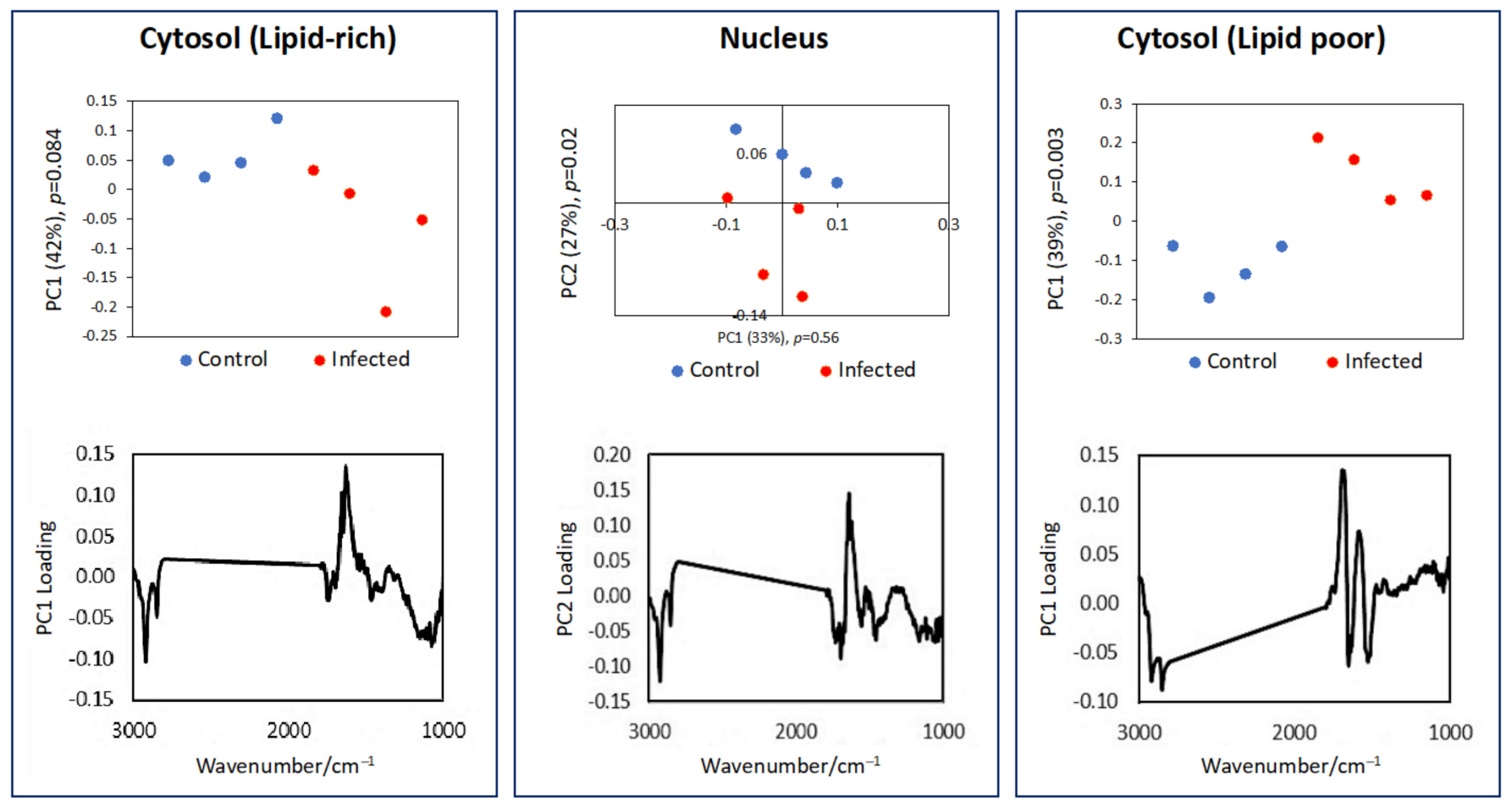

Figure 4. Pairwised (control versus infected) PCA of the extracted spectra (shown in Figure S5) from the three subcellular regions (cytosol (lipid-rich), nucleus, and cytosol (lipid poor)) with the most distinct differences between infected and uninfected cells at $24 \mathrm{hpi}$.
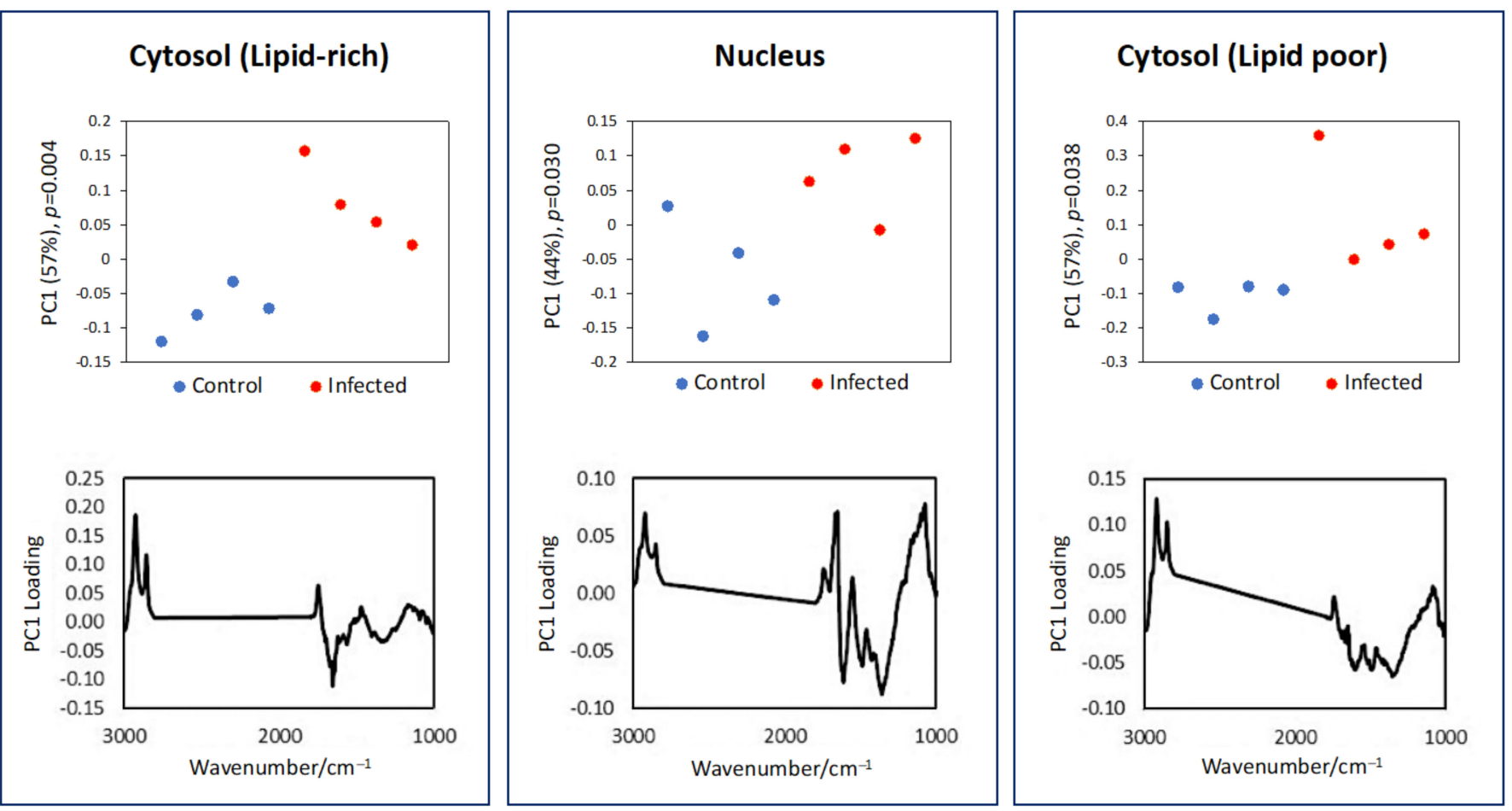

Figure 5. Pairwised (control versus infected) PCA1 of the extracted spectra (shown in Figure S6) from the three subcellular regions (cytosol (lipid-rich), nucleus, and cytosol (lipid poor)) with the most distinct differences between infected and uninfected cells at $48 \mathrm{hpi}$. 


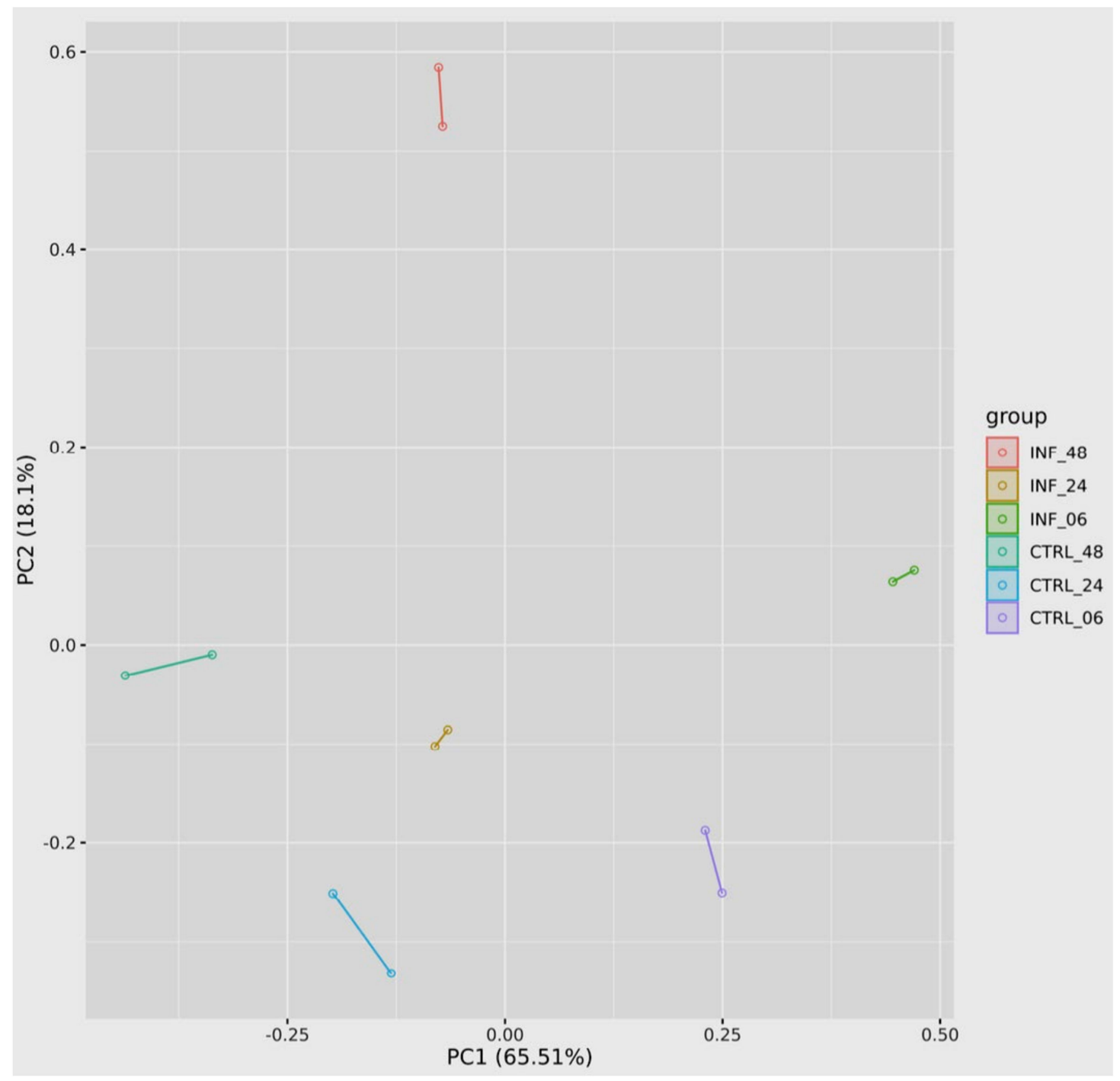

Figure 6. Principal Component Analysis of RNA-seq-based differential gene expression between infected and control hBMECs at 6-, 24- and 48-hpi. Two biological replicates are shown for each time point.

\subsection{Global Transcriptomic Changes}

RNA sequencing analysis of T. gondii-infected and uninfected hBMECs was performed to obtain more insight into the host-parasite relationship with a focus on genes that contribute to the mechanisms of metabolic adaptation and immune response of the host cell to T. gondii infection. The overall expression pattern of the DEGs at 6-, 24-, and 48-hpi was visualized using a PCA plot, which shows the variation caused by infection. Comparing control and infected cells at the same time point showed that the largest distance was detected between control and infected samples at $48 \mathrm{hpi}$, while the shortest distance was observed between control and infected samples at $24 \mathrm{hpi} \mathrm{(Figure} \mathrm{6).} \mathrm{RNA-seq} \mathrm{data}$ revealed 533 DEGs in the infected compared to uninfected hBMECs, of which 318 genes were upregulated and 215 genes were downregulated. These transcriptomic differences are clearly shown in the heatmap (Figure 7). The dendrogram on top of the heatmap shows the clustering of the samples based on gene expression similarities at 6-, 24-, and 48-hpi. Of the 533 DEGs, only five genes were differentially regulated over the entire infection time course. Of these, four genes (CHAC1, DDIT4, RPL37AP1, and MT-CO2) were upregulated and one gene (AHNAK2) was down-regulated (Table S1). ChaC glutathione- 
specific $\gamma$-glutamylcyclotransferase $1(C H A C 1)$ plays a role in regulating the inflammatory process. DNA damage-inducible transcript 4 (DDIT4) is activated under various cellular stresses, including DNA damage, hypoxia, oxidative stress, and starvation. DDIT4 also contributes to the regulation of various cell functions, including proliferation, apoptosis, and differentiation. RPL37AP1 (ribosomal protein L37a pseudogene 1), encodes ribosomal protein L37a, a component of 60S large ribosomal subunit, which is involved in protein synthesis from messenger RNAs. The mitochondrially encoded cytochrome c oxidase II (MT-CO2) is a component of respiratory complex IV.

Reactome pathway analysis of all DEGs identified 916 significant pathways (FDR < 0.05), 306 of these were downregulated. The top 30 most significantly enriched pathways are shown (Figure 8). The heatmap highlighted interchangeable expression in immune related signaling (i.e., interleukin IL-6 signaling) at different time points after infection. Out of the top 30 most different pathways, 10 pathways were common between 24 and 48 hpi samples, including ADORA2B-mediated anti-inflammatory cytokine production (R_HSA_9660821), CD163 mediating an anti-inflammatory response (R_HSA_9662834), cysteine formation from homocysteine (R_HSA_1614603), interleukin 10 signaling (R_HSA_6783783), interleukin 6 family signaling (R_HSA_6783589), interleukin 6 signaling (R_HSA_1059683), Leishmania parasite growth and survival (R_HSA_9664433), MAPK1 (ERK2) activation (R_HSA_112411), MAPK3 (ERK1) activation (R_HSA_110056), and sulfur amino acid metabolism (R_HSA_1614635).

At 6 hpi, 97 DEGs were identified, 23 of these were up-regulated and 74 were downregulated (Table S2). The three most up-regulated genes were CHAC1 (ENSG00000128965, ChaC glutathione specific gamma-glutamylcyclotransferase 1), PLCB1 (ENSG0000018261, phospholipase C beta 1), and DDIT4 (ENSG00000168209, DNA damage inducible transcript 4) with $\log _{2} \mathrm{FC}$ of $2.21,1.70$, and 1.69, respectively. The three most down-regulated genes included a novel gene (ENSG00000281181), RN7SL1 (ENSG00000276168, RNA component of signal recognition particle 7SL1), and TLN1 (ENSG00000137076, talin 1) with $\log _{2}$ FC of $-3.52,-2.49$, and -2.05 , respectively. The volcano plot (Figure 9) shows more downregulated $\left(\log _{2} \mathrm{FC}<-1\right.$; FDR $\left.<0.05\right)$ than up-regulated $\left(\log _{2} \mathrm{FC}>1\right.$; FDR $\left.<0.05\right)$ genes The heatmap of the top 30 DEGs is shown (Figure 10) with more genes down-regulated than up-regulated in the infected samples. To investigate the biological function of the DEGs induced by T. gondii at $6 \mathrm{hpi}$, the gene list in Table S2 with their expression values from edgeR counting were submitted to the REACTOME database for ssGSEA analysis to derive pathway expression values for every sample. The top 30 most significantly enriched pathways (between control and infected samples) are shown in the heatmap (Figure S7).

At 24 hpi, 32 DEGs were identified, of these 29 were up-regulated and 3 were downregulated (Table S3). The three most up-regulated genes included DHRS2 (ENSG00000100867, dehydrogenase/reductase 2), CHAC1 (ENSG00000128965, ChaC glutathione specific gammaglutamylcyclotransferase 1), and GABRG1 (ENSG00000163285, gamma-aminobutyric acid type A receptor subunit gamma1) with $\log _{2} \mathrm{FC}$ of $4.65,2.49$, and 2.44 , respectively. Only three down-regulated genes were detected, including PPL (ENSG00000118898, periplakin), AHNAK2 (ENSG00000185567, AHNAK nucleoprotein 2), and CCBE1 (ENSG00000183287, collagen and calcium binding EGF domains 1) with $\log _{2} \mathrm{FC}-1.37,-1.01$, and -1.01, respectively. The volcano plot shows more up-regulated genes than down-regulated genes (Figure 11). The heatmap of the top 30 DEGs is shown (Figure 12) with more genes upregulated than down-regulated in infected samples. The top 30 most significantly enriched pathways are shown in Figure S8.

At 48 hpi, 404 DEGs were identified, of these 266 were up-regulated and 138 were down-regulated (Table S4). The three most up-regulated genes were NR4A3 (ENSG00000119508, nuclear receptor subfamily 4 group A member 3), CXCL8 (ENSG00000169429, C-X-C motif chemokine ligand 8), and MT-RNR2 (ENSG00000210082, (mitochondrially encoded 16S rRNA) with $\log _{2} \mathrm{FC}$ of $4.89,4.59$, and 4.54 , respectively. $N R 4 A 3$ is a member of nuclear receptor subfamily 4 , which is an important regulator of cellular function and inflammatory signaling. The chemotactic pro-inflammatory cytokine interleukin-8 (CXCL8) plays a role 
in host response to intracellular pathogens. The MT-RNR2 (16S RNA) is a mitochondrialbased gene which encodes one rRNA subunit of mitochondrial ribosomes. The three most down-regulated genes are TMCC2 (ENSG00000133069, transmembrane and coiledcoil domain family 2), ZNF101 (ENSG00000181896, zinc finger protein 101), and LCA5 (ENSG00000135338, lebercilin LCA5), with $\log _{2}$ FC of $-8.99,-3.65$, and -3.55 , respectively. The volcano plot shows more up-regulated genes than down-regulated genes (Figure 13). The top 30 DEGs are shown in heatmap (Figure 14), where all genes were up-regulated in infected samples. The top 30 most significantly enriched pathways are shown in the heatmap (Figure S9).

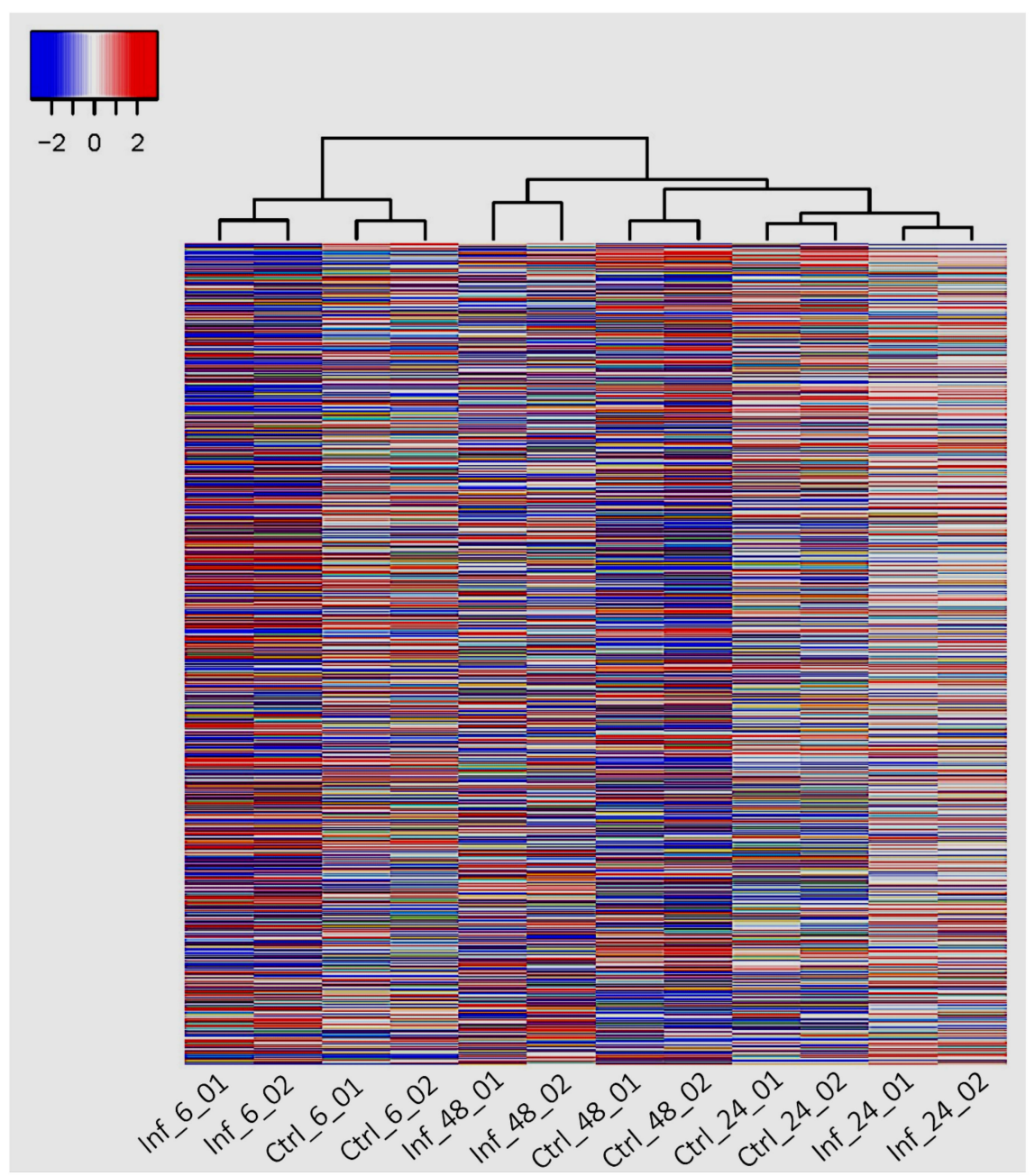

Figure 7. Heatmap showing hierarchical clustering of 533 DEGs at 6-, 24-, and 48-hpi. Only genes with $\log _{2} \mathrm{FC}$ of $<1$ or $>1$ and FDR $<0.05$ were included in the heatmap. The color scale from blue (low) to red (high) corresponds to the gene expression values. Columns represent the samples (infected and control) and time points, while rows represent individual genes. The phylogenetic tree above the heatmap shows the hierarchical clustering of the samples. 


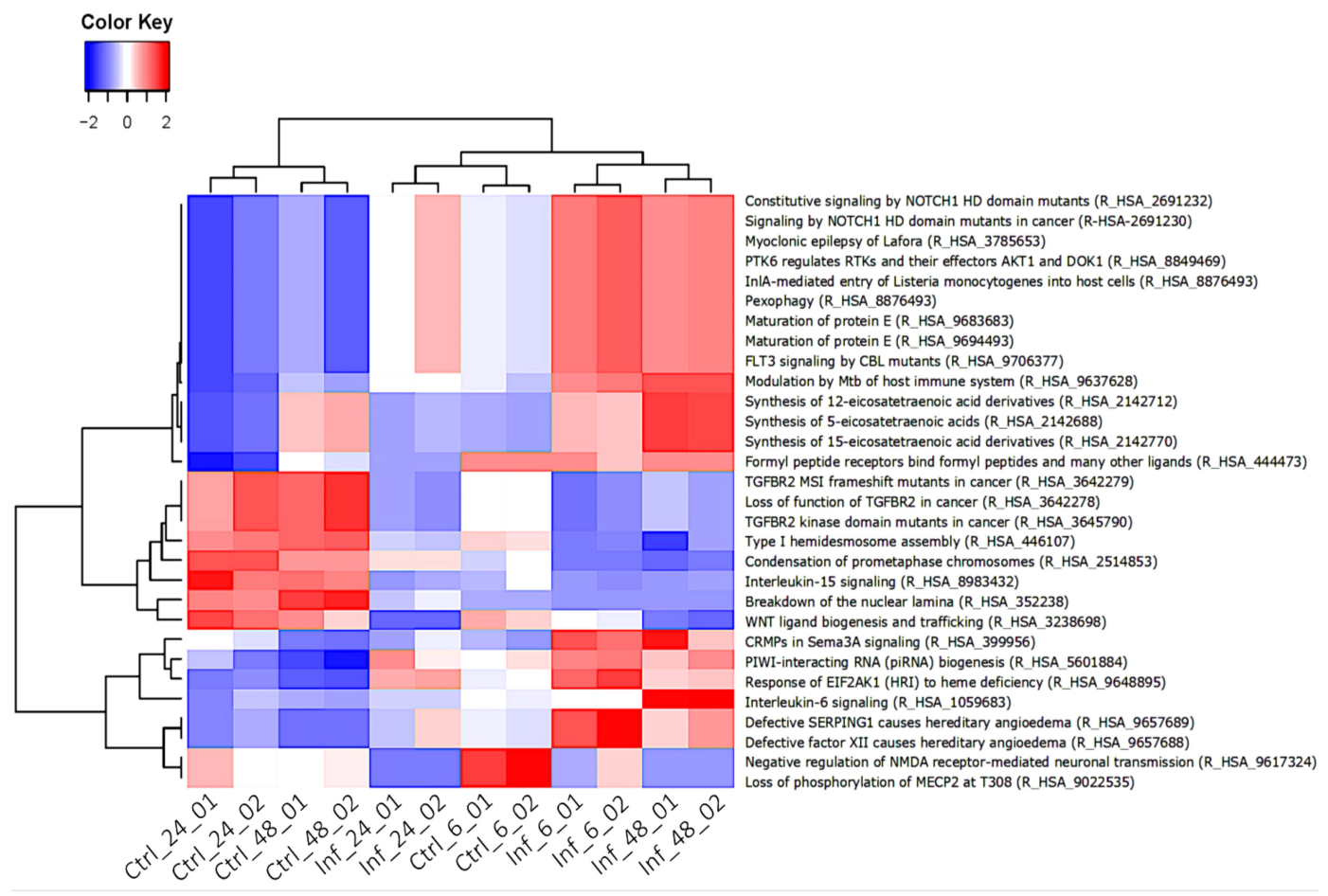

Figure 8. Heatmap clustering representing of the top 30 pathways $(\mathrm{FDR}<0.05)$ in all samples. The color scale from blue (low) to red (high) corresponds to the expression values. The pathway name and Reactome pathway ID are shown on the right side of the heatmap. Columns represent the samples and time points post infection, while rows represent individual pathways. The phylogenetic tree above the heatmap shows the hierarchical clustering of the samples. The relationships between the pathways are shown on the left tree.

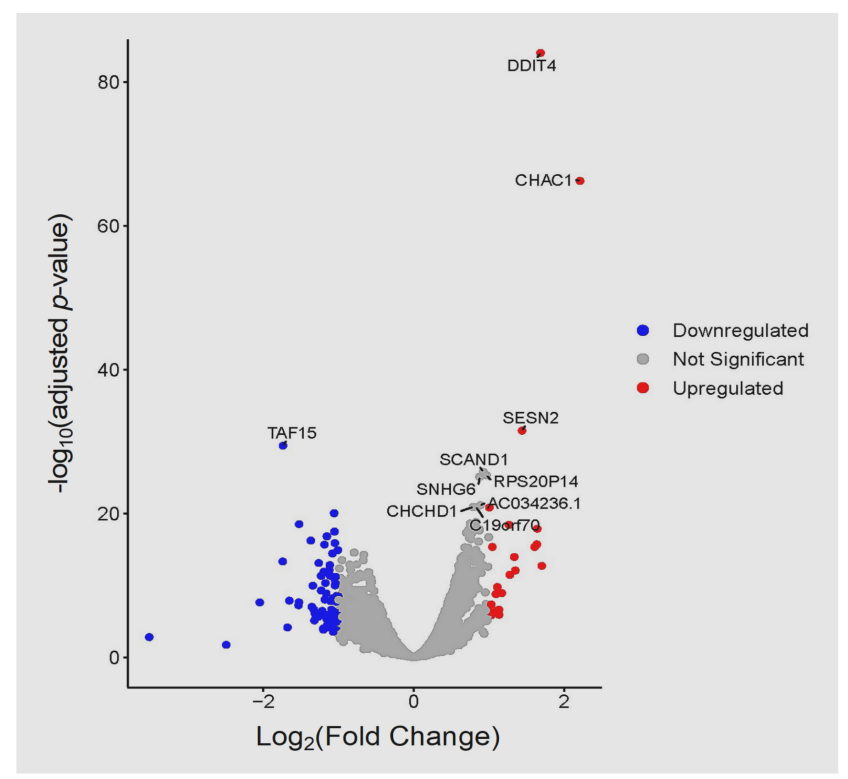

Figure 9. Volcano plot comparing the top 10 DEGs, with the highest differences in expression, between infected and control samples at 6 hpi. Upregulated (red) and downregulated (blue) genes are shown. The fold-changes in gene expression between samples $\left(\log _{2} \mathrm{FC}\right)$ are plotted on the $x$-axis and the $y$-axis shows statistical significance of the differences ( $p$-values). Each dot in the plot represents one gene. 


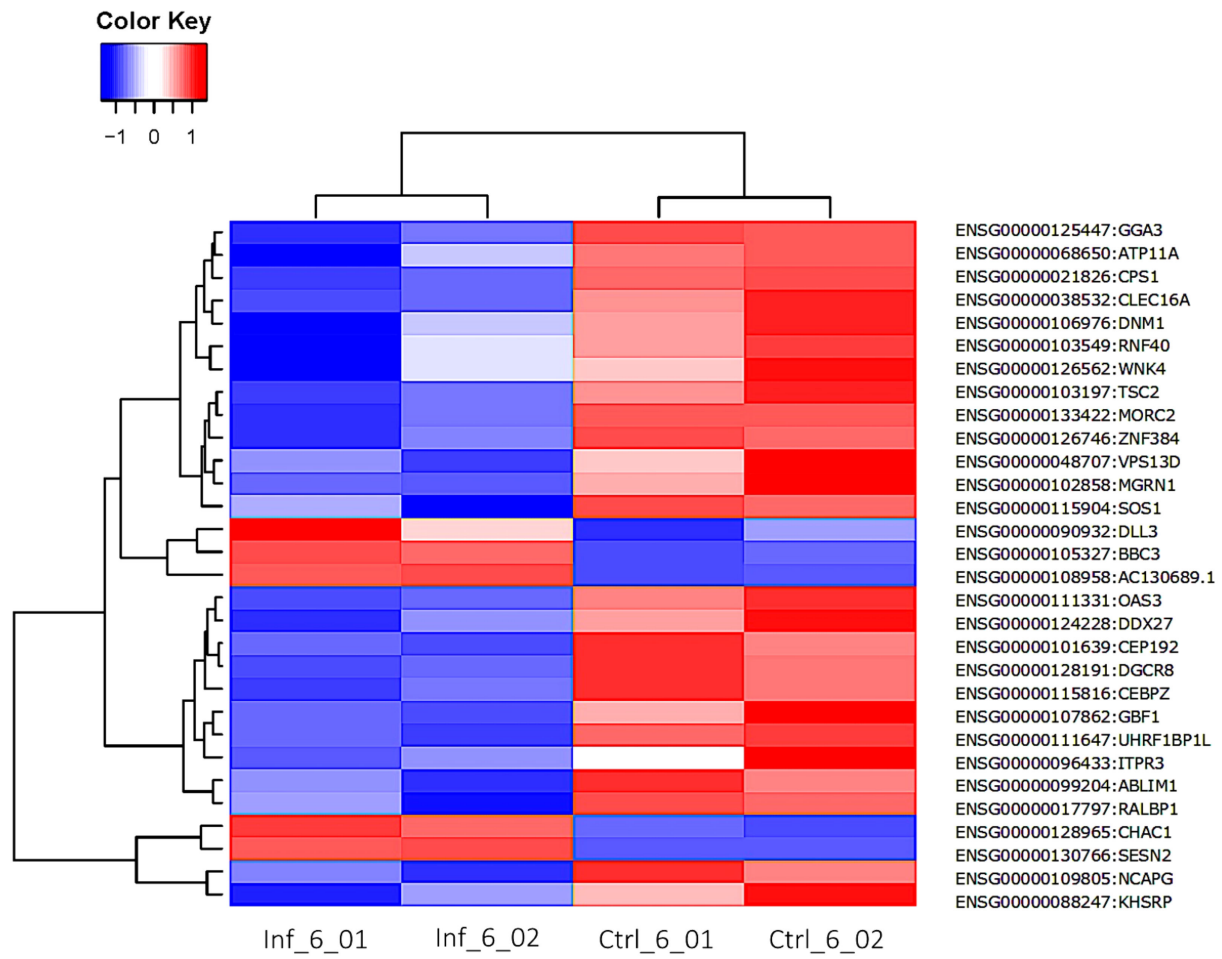

Figure 10. Heatmap generated from the $\log _{2} \mathrm{FC}$ of the top 30 DEGs at 6 hpi. Blue and red colors indicate low and high gene expression, respectively. Gene IDs are indicated on the right side of the heatmap. Columns represent the samples and time points post infection, while rows represent individual genes. The phylogenetic tree above the heatmap shows the hierarchical clustering of the samples. The relationships between the genes are shown on the left tree. For each gene symbol, the respective $\log _{2} \mathrm{FC}$ and other information can be found in Table S2.

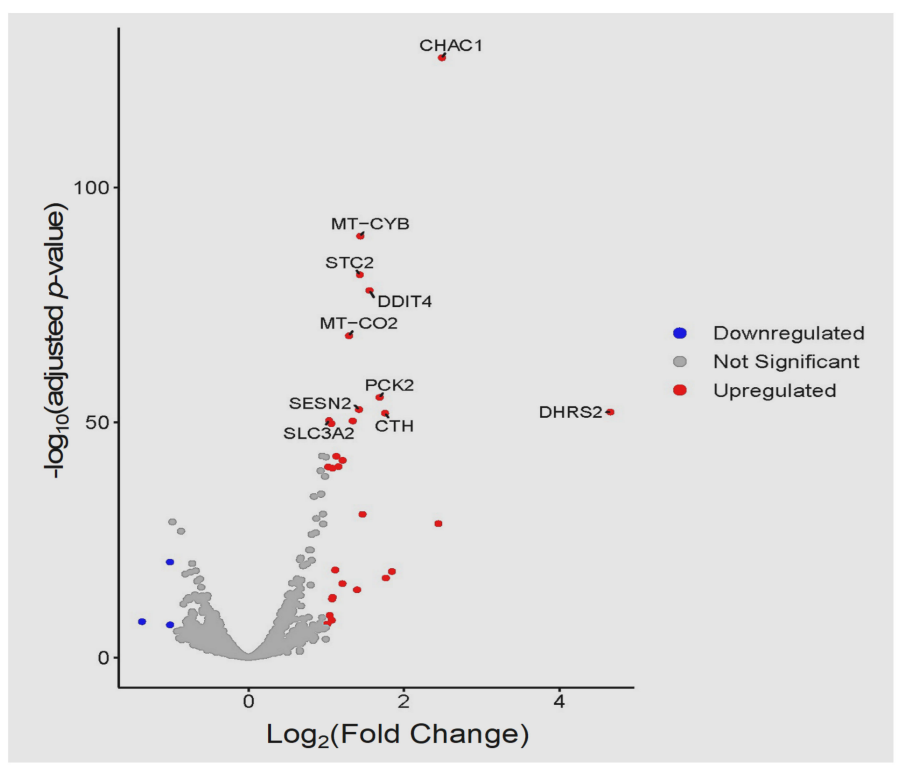

Figure 11. Volcano plot comparing the top 10 DEGs, with the highest differences in expression, between infected and control samples at 24 hpi. Upregulated (red) and downregulated (blue) genes are shown. $p$-values were plotted on the $y$-axis while $\log _{2} \mathrm{FC}$ values are plotted on the $x$-axis. Each dot in the plot represents one gene. 


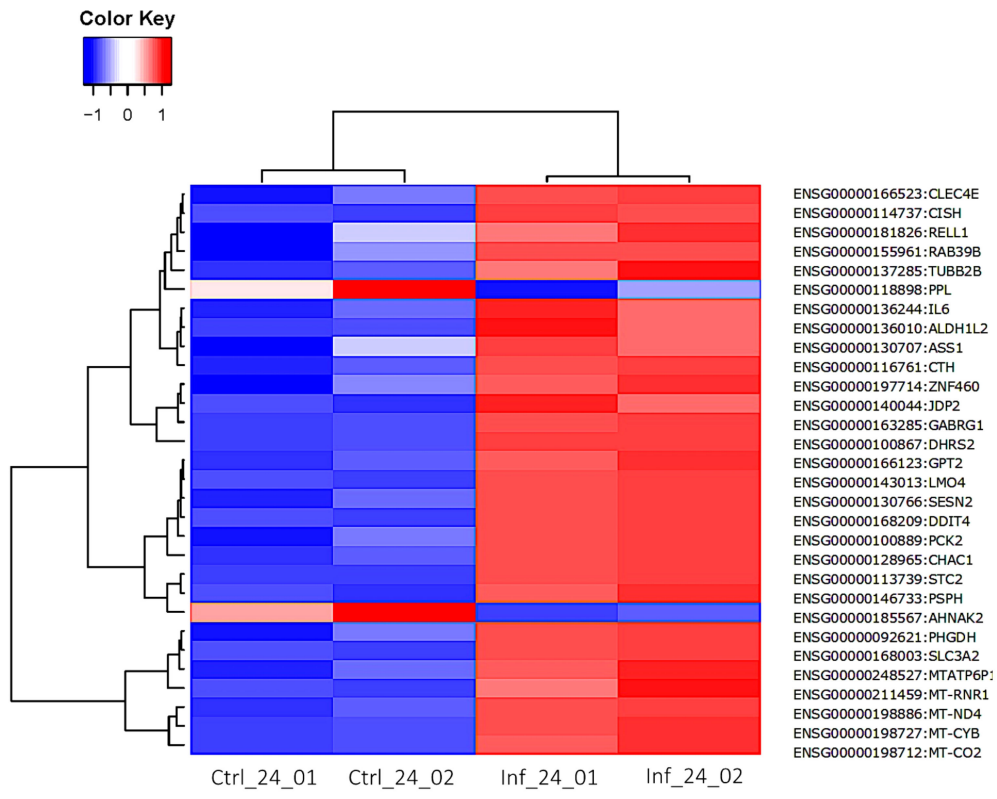

Figure 12. Heatmap generated from the $\log _{2} \mathrm{FC}$ of the top $30 \mathrm{DEGs}$ at 24 hpi. Blue and red colors indicate low and high gene expression, respectively. Gene IDs are indicated on the right side of the heatmap. Columns represent the samples and time points post infection, while rows represent individual genes. The phylogenetic tree above the heatmap demonstrates the hierarchical clustering of the samples. The relationships between the genes are shown on the left tree. For each gene symbol, the respective $\log 2 \mathrm{FC}$ and other information can be found in Table S3.

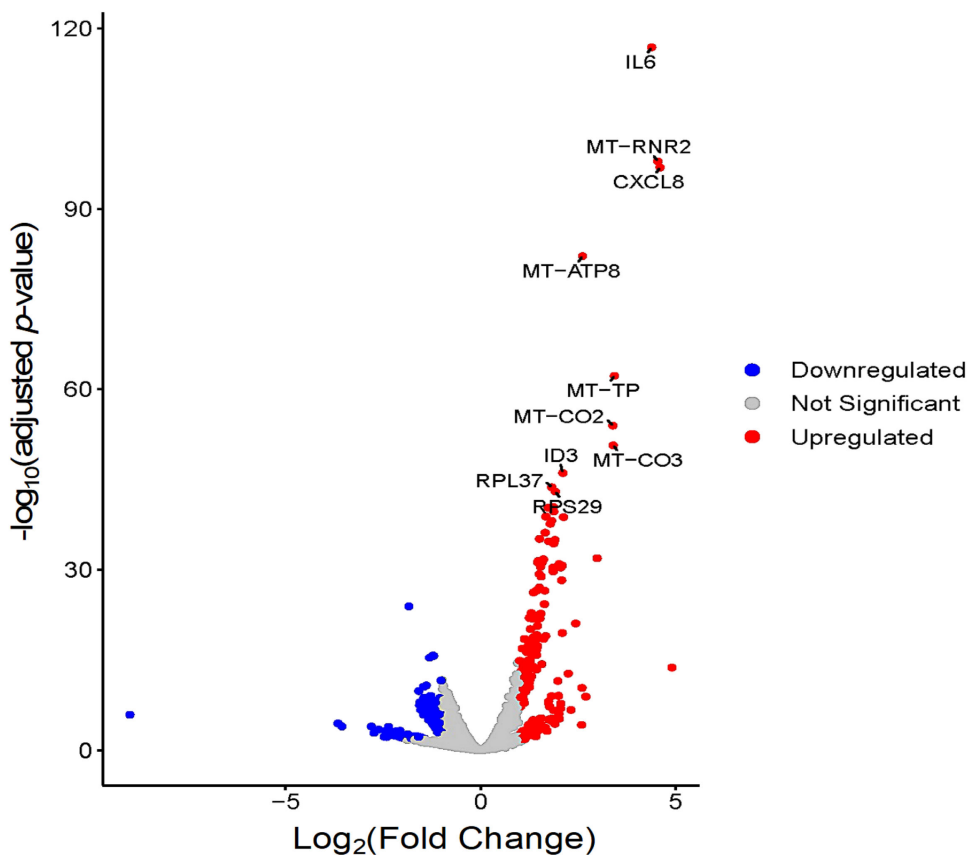

Figure 13. Volcano plot comparing the top 10 genes with the highest differences in expression between infected and control samples at $48 \mathrm{hpi}$. Upregulated (red) and downregulated (blue) genes are shown. $p$-values were plotted on the $y$-axis while $\log _{2} \mathrm{FC}$ values are plotted on the $x$-axis. Each dot in the plot represents one gene. 


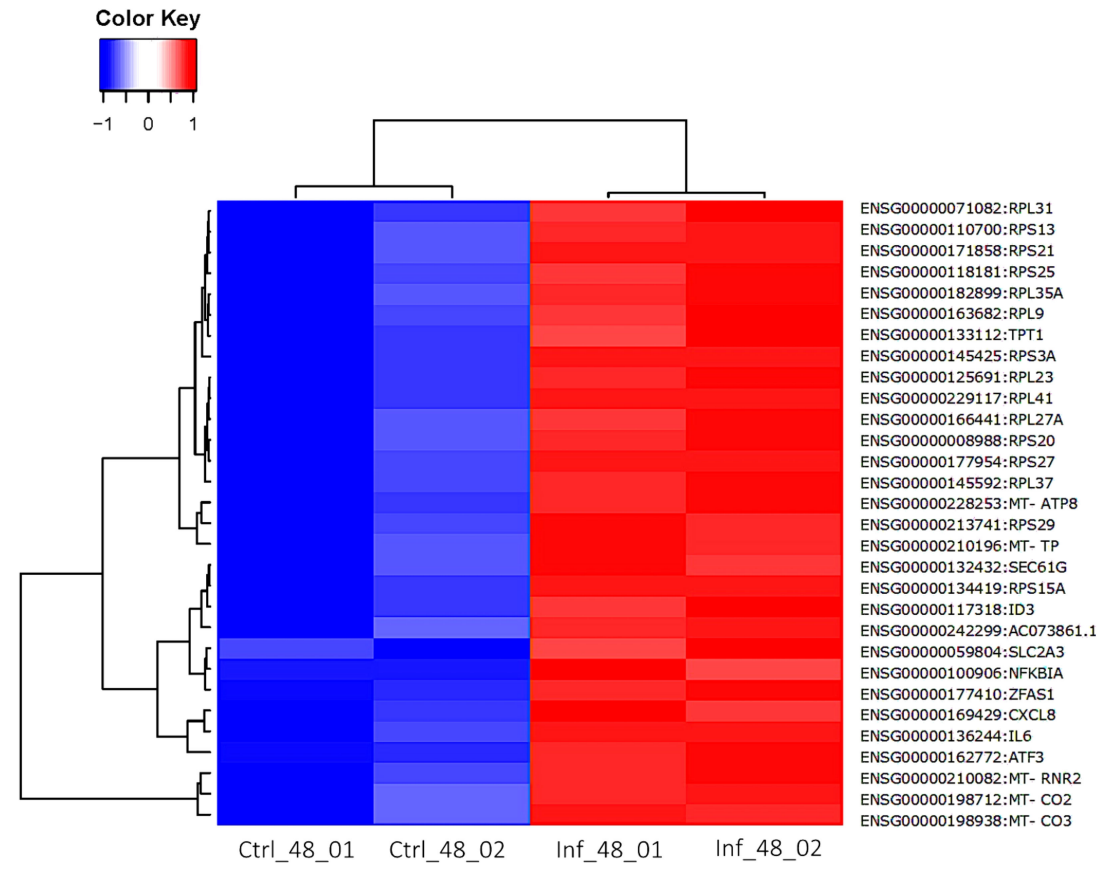

Figure 14. Heatmap generated from the $\log _{2} \mathrm{FC}$ of the top 30 DEGs at 48 hpi. Blue and red colors indicate low and high gene expression, respectively. Gene IDs are indicated on the right side of the heatmap. Columns represent the samples and time points post infection, while rows represent individual genes. The phylogenetic tree above the heatmap demonstrates the hierarchical clustering of the samples. The relationships between the genes are shown on the left tree. For each gene symbol, the respective $\log _{2}$ FC and other information can be found in Table S4.

\section{Discussion}

Tracking the molecular changes of host cells in response to infection progression is fundamental for the understanding of host-pathogen interaction and the development of therapeutic interventions. Label-free spectroscopic imaging has been emerging as a central tool for elucidating the global chemical changes caused by infection with obligate intracellular protozoan parasites, such as T. gondii [4,9]. FTIR spectroscopy is an effective label-free imaging tool that can detect the (bio)molecular constituents of individual host cells without the use of fluorescent probes [27]. In the present study, we investigated the general dynamics of chemical changes that accompany T. gondii infection of hBMECs using conventional FTIR micro spectroscopic analysis, which revealed no significant differences between infected and control cells at $24 \mathrm{hpi}$ or $48 \mathrm{hpi}$ in PC1. This result suggests that spectral changes that are not specific to infection were strong despite the rigorous data pretreatment processes applied. This could also be due to lower spectral signal-to-noise ratio when compared to SR-FTIR or artefacts associated with the measurement of dried cells. However, significant differences were observed in PC2 with changes mainly associated with the lipid CH stretching bands and protein amide I and II. This result corroborates a previous study where clustering analysis of the FTIR spectral data showed an alteration in the lipid to protein ratio in the infected cells, which was confirmed by biochemical assays [2]. At $24 \mathrm{hpi}$, infected cells had an overall lower lipid content, however at $48 \mathrm{hpi}$, the lipid content was higher in the infected cells.

Although FTIR spectroscopy has been previously used to distinguish FTIR spectra of T. gondii-infected cells from those of uninfected cells [2], we argue that there is a need to dissociate the differences between infected and uninfected cells not only at the single cell level, but also at the sub-cellular scale. Therefore, we used synchrotron based FTIR microspectroscopy to investigate whether FTIR spectral data can be used to study T. gondii infection-driven chemical alterations at the sub-cellular level. SR-FTIR ZnS hemisphere analysis showed that infected cells had a lower lipid content in the cytosol region of infected 
cells at 24 hpi. The overall lipid content was however higher in the infected cells at 48 hpi, where PCA of all three subcellular regions with the most distinct differences (i.e., lipid-rich cytosol, nucleus, or lipid-poor cytosol) showed a higher lipid content. In the lipid poor region of the cytosol, PC1 has shown a significant difference between control and infected cells with changes mainly found in the protein amide I and II peaks. These changes are consistent with the findings obtained by conventional FTIR analysis.

Alterations in the lipid and protein contents of hBMECs have been previously detected using proton nuclear magnetic resonance-based metabolomic analysis [7,9]. In addition, changes in the abundance of lipids (e.g., glycerophospholipid) and amino acid (phenylalanine, retinol, and tryptophan) were detected in mouse cerebellum during T. gondii infection by using ultra performance liquid chromatography-tandem mass spectrometrybased metabolomics [28]. However, the application of these methods requires a special sample preparation, which may alter the biochemical composition of the host cells. In contrast, vibrational spectroscopy techniques, such as the FTIR used in the present study, can be applied in nondestructive and label-free analysis of biochemical changes in the host cells. The chemical changes in infected host cells can be significant enough to be inferred from the FTIR spectra at the single cell level, whereas SR-FTIR ZnS hemisphere analysis can provide insight into changes of the chemical composition of host cells at the sub-cellular level. The application of conventional FTIR and synchrotron FTIR clearly showed how these methods can detect infection-related molecular changes as early as 24 hpi. Importantly, these approaches can be applied for live-cell imaging, increasing the scope for further investigation of host cell-parasite dynamics. It is noteworthy to mention that conventional micro FTIR was used for imaging whole cells while SR-based FTIR offered subcellular spatial resolution, which complicates direct quantitative comparisons of spectra obtained by the two imaging approaches.

RNA-seq, a high-throughput method for characterizing transcriptomes, was used to compare the transcription levels of genes between T. gondii-infected and control hBMECs at 6, 24 and $48 \mathrm{hpi}$. We hypothesized that global profiling of transcriptional changes in T. gondii-infected versus uninfected cells could elucidate the metabolic and regulatory mechanisms involved in host cell response to T. gondii infection. T. gondii infection altered the expression of genes involved in cellular processes and regulatory events, such as immune signaling, adaptation to stress, and amino acid metabolism. Sulphur amino acid metabolism was one of the pathways commonly found between 24 and 48 hpi samples. This pathway can be modulated by T. gondii protein (elongation factor 1-alpha, putative; (EF-1-alpha). Immunization of mice using EF-1-alpha-based monoclonal antibody vaccine increased survival of mice against $T$. gondii infection, suggesting the immunogenicity of EF-1-alpha and its involvement in mediating host-T. gondii interaction in mice [29]. What roles other T. gondii effector proteins play in the modulation of the host sulfur amino acid metabolism remain to be elucidated.

Pathway analysis also revealed that transcriptomic changes were predominantly part of a defense mechanism involving key cytokines (e.g., IL-6, IL-8, IL-10). IL-6 and IL-8 are involved in the development of protective immunity against T. gondii, whereas IL-10 is required to limit T. gondii infection-induced pathology. This immune-balance could be an advantage for $T$. gondii if a large proportion of host cell gene expression is directed at immune responses, and T. gondii already possesses mechanisms for countering these. Other host processes may then be easier to interfere with and hijacked by $T$. gondii. The results of the present and previous studies [30-35], confirm the ability of T. gondii to manipulate various cellular processes, particularly immune response via reprogramming host gene expression to support its own survival within the host cells.

\section{Conclusions}

We have investigated the molecular mechanisms underlying the interactions of T. gondii with its host cell at the biochemical and transcriptomic level, which is the first characterization of the dynamics of T. gondii infection at this level of detail. We applied conventional 
and synchrotron based FTIR microspectroscopy to elucidate the chemical changes that are associated with T. gondii infection of hBMECs. We studied how the lipid, protein, and nucleic acid compositions change after exposure to infection using information from the FTIR spectra. Our results showed that FTIR spectroscopy delivers the necessary resolution to uncover chemical alterations within individual cells and at the sub-cellular level. Conventional FTIR analysis and SR-FTIR ZnS hemisphere approach showed that the lipid content of infected cells was decreased at $24 \mathrm{hpi}$ but increased at $48 \mathrm{hpi}$. These label free imaging methods improved our knowledge of the cellular processes taking place during T. gondii infection. Transcriptomic analysis revealed genes and signaling pathways that play various roles in mediating the host cell response to T. gondii infection. To determine the involvement of specific molecular species will require the integration of these chemical imaging tools with targeted analytical approaches, such as isotope labeling methods and substrate enrichments, and the further investigation of how the host cells respond and adapt to T. gondii infection.

Supplementary Materials: The following are available online at https:/ /www.mdpi.com/article/10 .3390 / cells11050811/s1, Figure S1: FTIR images (integration range shown on the left column) of the three other host cells at 24 hpi. Scale bars $=10 \mu \mathrm{m}$, Figure S2: FTIR images (integration range shown on the left column) of the three other control cells at $24 \mathrm{~h}$. The visible images were not collected for two cells. Scale bars $=10 \mu \mathrm{m}$, Figure S3: FTIR images (integration range shown on the left column) of the three other cells at $48 \mathrm{hpi}$. Scale bars $=10 \mu \mathrm{m}$, Figure S4: FTIR images (integration range shown on the left column) of the three other control cells at $48 \mathrm{~h}$. Scale bars $=10 \mu \mathrm{m}$, Figure S5: The extracted spectra of infected and control cells at 24 hpi from the lipid-rich, DNA-rich, and lipid-poor regions, Figure S6: The extracted spectra of infected and control cells at 48 hpi from the lipid-rich, DNA-rich, and lipid-poor regions, Figure S7: The top 30 pathways most significantly activated (red) or inactivated (blue) at $6 \mathrm{hpi}$ by T. gondii. Columns represent replicates of the samples and rows represent individual pathways. The phylogenetic tree above the heatmap demonstrates the hierarchical clustering of the samples. The relationships between the pathways are shown on the left tree, Figure S8: The top 30 pathways most significantly activated (red) or inactivated (blue) at 24 hpi by T. gondii. Columns represent replicates of the samples and rows represent individual pathways. The phylogenetic tree above the heatmap demonstrates the hierarchical clustering of the samples. The relationships between the pathways are shown on the left tree, Figure S9: The top 30 pathways most significantly activated (red) or inactivated (blue) at 48 hpi by $T$. gondii. Columns represent replicates of the samples and rows represent individual pathways. The phylogenetic tree above the heatmap demonstrates the hierarchical clustering of the samples. The relationships between the pathways are shown on the left tree, Table S1: Differentially expressed genes commonly detected at 6-, 24-, and 48-hpi by T. gondii, Table S2: List of the 97 differentially expressed genes (23 upregulated and 74 downregulated) with FDR $<0.05$ and absolute $\log _{2} \mathrm{FC}<-1$ and $>1$ at 6 hpi. The respective gene identifier, gene symbol, and $\log _{2} \mathrm{FC}$ are shown, Table S3: List of the 32 differentially expressed genes (29 up-regulated and 3 down-regulated) with FDR $<0.05$ and absolute $\log _{2} \mathrm{FC}<-1$ and $>1$ at $24 \mathrm{hpi}$. The respective gene identifier, gene symbol, and $\log _{2}$ FC are shown, Table S4: List of the 404 differentially expressed genes (266 up-regulated and 138 down-regulated) with FDR $<0.05$ and absolute $\log _{2} \mathrm{FC}<-1$ and $>1$ at 48 hpi. The respective gene identifier, gene symbol, and $\log _{2} \mathrm{FC}$ are shown.

Author Contributions: Conceptualization, H.M.E. and K.L.A.C.; methodology, H.M.E. and K.L.A.C.; software, G.C., M.S.R.H., H.M.E. and K.L.A.C.; validation, A.T.A.-S., W.M. and H.M.E.; formal analysis, A.T.A.-S., M.S.R.H., F.W., K.L.A.C. and H.M.E.; investigation, A.T.A.-S., A.A. and F.W.; resources, M.S.R.H., N.A.E., A.A., W.M., J.G.M.M., P.W.D., K.L.A.C. and G.C.; data curation, H.M.E. and K.L.A.C.; writing-original draft preparation, H.M.E. and K.L.A.C.; writing-review and editing, H.M.E., N.A.E., W.M., J.G.M.M., P.W.D., C.W.S., G.C. and K.L.A.C.; visualization, H.M.E. and K.L.A.C.; supervision, H.M.E., K.L.A.C. and C.W.S.; project administration, H.M.E.; funding acquisition, N.A.E., A.T.A.-S., F.W. and H.M.E. All authors have read and agreed to the published version of the manuscript. 
Funding: A.T.A.-S. was supported by a Ph.D. studentship from the Ministry of Higher Education of the Iraqi government. F.D. was supported by a Wellcome Trust CVRT Vacation Award. N.A.E. was supported by University of Nottingham Vice-Chancellor Scholarship for international students. The study was partly supported by a BBSRC grant BB/M024156/1 to P.W.D.

Institutional Review Board Statement: Not applicable.

Informed Consent Statement: Not applicable.

Data Availability Statement: The RNA sequencing reads have been deposited in the ArrayExpress of the European Bioinformatics Institute under accession no. E-MTAB-11515.

Acknowledgments: The authors would like to acknowledge the UK's National Synchrotron Science facility, Diamond Light Source for the provision of beamtime at B22 provided under proposal [SM20222-1]

Conflicts of Interest: The authors declare no conflict of interest. The funders had no role in the design of the study; in the collection, analyses, or interpretation of data; in the writing of the manuscript, or in the decision to publish the results.

\section{References}

1. Hill, D.E.; Chirukandoth, S.; Dubey, J.P. Biology and epidemiology of Toxoplasma gondii in man and animals. Anim. Health Res. Rev. 2005, 6, 41-61. [CrossRef]

2. Elsheikha, H.M. Congenital toxoplasmosis: Priorities for further health promotion action. Public Health 2008, 122, 335-353. [CrossRef]

3. Elsheikha, H.M.; Marra, C.M.; Zhu, X.Q. Epidemiology, pathophysiology, diagnosis, and management of cerebral toxoplasmosis. Clin. Microbiol. Rev. 2021, 34, e00115-19. [CrossRef]

4. Naemat, A.; Elsheikha, H.M.; Al-Sandaqchi, A.; Kong, K.; Ghita, A.; Notingher, I. Analysis of interaction between the apicomplexan protozoan Toxoplasma gondii and host cells using label-free Raman spectroscopy. Analyst 2015, 140, 756-764. [CrossRef]

5. Ma, J.; He, J.J.; Hou, J.L.; Zhou, C.X.; Zhang, F.K.; Elsheikha, H.M.; Zhu, X.Q. Metabolomic signature of mouse cerebral cortex following Toxoplasma gondii infection. Parasit. Vectors 2019, 12, 373. [CrossRef]

6. Zhou, C.X.; Zhou, D.H.; Elsheikha, H.M.; Liu, G.X.; Suo, X.; Zhu, X.Q. Global metabolomic profiling of Mice brains following experimental infection with the cyst-forming Toxoplasma gondii. PLoS ONE 2015, 10, e0139635. [CrossRef]

7. Al-Sandaqchi, A.T.; Marsh, V.; Williams, H.E.L.; Stevenson, C.W.; Elsheikha, H.M. Structural, functional, and metabolic alterations in human cerebrovascular endothelial cells during Toxoplasma gondii infection and amelioration by verapamil in vitro. Microorganisms 2020, 8, 1386. [CrossRef]

8. Kendall, C.; Isabelle, M.; Bazant-Hegemark, F.; Hutchings, J.; Orr, L.; Babrah, J.; Baker, R.; Stone, N. Vibrational spectroscopy: A clinical tool for cancer diagnostics. Analyst 2009, 134, 1029-1045. [CrossRef]

9. Elsheikha, H.M.; Elsaied, N.A.; Chan, K.L.A.; Brignell, C.; Harun, M.S.R.; Wehbe, K.; Cinque, G. Label-free characterization of biochemical changes within human cells under parasite attack using synchrotron based micro-FTIR. Anal. Methods 2019, 11, 2518-2530. [CrossRef]

10. Wrobel, T.P.; Bhargava, R. Infrared spectroscopic imaging advances as an analytical technology for biomedical sciences. Anal. Chem. 2018, 90, 1444-1463. [CrossRef]

11. Wrobel, T.P.; Marzec, K.M.; Majzner, K.; Kochan, K.; Bartus, M.; Chlopicki, S.; Baranska, M. Attenuated total reflection Fourier transform infrared (ATR-FTIR) spectroscopy of a single endothelial cell. Analyst 2012, 137, 4135-4139. [CrossRef]

12. Chan, K.L.A.; Fale, P.L.V.; Atharawi, A.; Wehbe, K.; Cinque, G. Subcellular mapping of living cells via synchrotron microFTIR and ZnS hemispheres. Anal. Bioanal. Chem. 2018, 410, 6477-6487. [CrossRef]

13. Chan, K.L.A.; Kazarian, S.G. Correcting the effect of refraction and dispersion of light in FT-IR spectroscopic imaging in transmission through thick infrared windows. Anal. Chem. 2013, 85, 1029-1036. [CrossRef]

14. Chan, K.L.A.; Kazarian, S.G. Aberration-free FTIR spectroscopic imaging of live cells in microfluidic devices. Analyst 2013, 138, 4040-4047. [CrossRef]

15. Chan, K.L.A.; Altharawi, A.; Fale, P.; Song, C.L.; Kazarian, S.G.; Cinque, G.; Untereiner, V.; Sockalingum, G.D. Transmission Fourier transform infrared spectroscopic imaging, mapping, and synchrotron scanning microscopy with zinc sulfide hemispheres on living mammalian cells at sub-cellular resolution. Appl. Spectrosc. 2020, 74, 544-552. [CrossRef]

16. Elsheikha, H.M.; McKinlay, C.L.; Elsaied, N.A.; Smith, P.A. Effects of Neospora caninum infection on brain microvascular endothelial cells bioenergetics. Parasit. Vectors 2013, 6, 24. [CrossRef]

17. Elsheikha, H.M.; Alkurashi, M.; Kong, K.; Zhu, X.Q. Metabolic footprinting of extracellular metabolites of brain endothelium infected with Neospora caninum in vitro. BMC Res. Notes 2014, 7, 406. [CrossRef]

18. Al-Sandaqchi, A.T.; Brignell, C.; Collingwood, J.F.; Geraki, K.; Mirkes, E.M.; Kong, K.; Castellanos, M.; May, S.T.; Stevenson, C.W.; Elsheikha, H.M. Metallome of cerebrovascular endothelial cells infected with Toxoplasma gondii using mu-XRF imaging and inductively coupled plasma mass spectrometry. Metallomics 2018, 10, 1401-1414. [CrossRef] 
19. Elsheikha, H.M.; Alkurashi, M.; Palfreman, S.; Castellanos, M.; Kong, K.; Ning, E.; Elsaied, N.A.; Geraki, K.; MacNaughtan, W. Impact of Neospora caninum infection on the bioenergetics and transcriptome of cerebrovascular endothelial cells. Pathogens 2020, 9, 710. [CrossRef]

20. Harun, M.S.R.; Taylor, M.; Zhu, X.Q.; Elsheikha, H.M. Transcriptome profiling of Toxoplasma gondii-infected human cerebromicrovascular endothelial cell response to treatment with monensin. Microorganisms 2020, 8, 842. [CrossRef]

21. Elsheikha, H.M.; Rosenthal, B.M.; Murphy, A.J.; Dunams, D.B.; Neelis, D.A.; Mansfield, L.S. Generally applicable methods to purify intracellular coccidia from cell cultures and to quantify purification efficacy using quantitative PCR. Vet. Parasitol. 2006, 135, 223-234. [CrossRef] [PubMed]

22. Jarvis, R.M.; Broadhurst, D.; Johnson, H.; O’Boyle, N.M.; Goodacre, R. PYCHEM: A multivariate analysis package for python. Bioinformatics 2006, 22, 2565-2566. [CrossRef] [PubMed]

23. Robinson, M.D.; McCarthy, D.J.; Smyth, G.K. edgeR: A Bioconductor package for differential expression analysis of digital gene expression data. Bioinformatics 2010, 26, 139-140. [CrossRef]

24. Griss, J.; Viteri, G.; Sidiropoulos, K.; Nguyen, V.; Fabregat, A.; Hermjakob, H. ReactomeGSA—Efficient multi-omics comparative pathway analysis. Mol. Cell. Proteom. 2020, 19, 2115-2125. [CrossRef]

25. Vongsvivut, J.; Perez-Guaita, D.; Wood, B.R.; Heraud, P.; Khambatta, K.; Hartnell, D.; Hackett, M.J.; Tobin, M.J. Synchrotron macro ATR-FTIR microspectroscopy for high-resolution chemical mapping of single cells. Analyst 2019, 144, 3226-3238. [CrossRef] [PubMed]

26. Whelan, D.R.; Bambery, K.R.; Heraud, P.; Tobin, M.J.; Diem, M.; McNaughton, D.; Wood, B.R. Monitoring the reversible B to A-like transition of DNA in eukaryotic cells using Fourier transform infrared spectroscopy. Nucleic Acids Res. 2011, 39, 5439-5448. [CrossRef]

27. Cinque, G.; Frodely, M.D.; Wehbe, K.; Que Nguyen, T.-N.; Fitzpatrick, A.; Kelley, C.S. Synchrotron-based infrared spectral imaging at the MIRIAM beamline of Diamond Light Source. Synchrotron Radiat. News 2017, 30, 11-16. [CrossRef]

28. Ma, J.; He, J.J.; Hou, J.L.; Zhou, C.X.; Elsheikha, H.M.; Zhu, X.Q. Ultra performance liquid chromatography-tandem mass spectrometry-based metabolomics reveals metabolic alterations in the mouse cerebellum during Toxoplasma gondii infection. Front. Microbiol. 2020, 11, 1555. [CrossRef]

29. Wang, S.; Zhang, Z.; Wang, Y.; Gadahi, J.A.; Xu, L.; Yan, R.; Song, X.; Li, X. Toxoplasma gondii elongation factor 1-alpha (TgEF-1alpha) is a novel vaccine candidate antigen against toxoplasmosis. Front. Microbiol. 2017, 8, 168. [CrossRef]

30. Li, J.X.; He, J.J.; Elsheikha, H.M.; Chen, D.; Zhai, B.T.; Zhu, X.Q.; Yan, H.K. Toxoplasma gondii ROP17 inhibits the innate immune response of HEK293T cells to promote its survival. Parasitol. Res. 2019, 118, 783-792. [CrossRef]

31. Hu, R.S.; He, J.J.; Elsheikha, H.M.; Zou, Y.; Ehsan, M.; Ma, Q.N.; Zhu, X.Q.; Cong, W. Transcriptomic profiling of mouse brain during acute and chronic infections by Toxoplasma gondii oocysts. Front. Microbiol. 2020, 11, 570903. [CrossRef]

32. He, J.J.; Ma, J.; Wang, J.L.; Zhang, F.K.; Li, J.X.; Zhai, B.T.; Wang, Z.X.; Elsheikha, H.M.; Zhu, X.Q. Global transcriptome profiling of multiple porcine organs reveals Toxoplasma gondii-induced transcriptional landscapes. Front. Immunol. 2019, 10, 1531. [CrossRef]

33. Li, J.X.; He, J.J.; Elsheikha, H.M.; Ma, J.; Xu, X.P.; Zhu, X.Q. ROP18-Mediated transcriptional reprogramming of HEK293T cell reveals new roles of ROP18 in the interplay between Toxoplasma gondii and the host cell. Front. Cell. Infect. Microbiol. 2020, 10, 586946. [CrossRef] [PubMed]

34. Zhu, Y.C.; Elsheikha, H.M.; Wang, J.H.; Fang, S.; He, J.J.; Zhu, X.Q.; Chen, J. Synergy between Toxoplasma gondii type I $\Delta$ GRA17 immunotherapy and PD-L1 checkpoint inhibition triggers the regression of targeted and distal tumors. J. Immunother. Cancer 2021, 9, e002970. [CrossRef] [PubMed]

35. He, J.J.; Ma, J.; Elsheikha, H.M.; Song, H.Q.; Huang, S.Y.; Zhu, X.Q. Transcriptomic analysis of mouse liver reveals a potential hepato-enteric pathogenic mechanism in acute Toxoplasma gondii infection. Parasit. Vectors 2016, 9, 427. [CrossRef] [PubMed] 